\title{
Design of spectral-spatial phase prewinding pulses and their use in small-tip fast recovery steady-state imaging.
}

\author{
Sydney N. Williams, Jon-Fredrik Nielsen, Jeffrey A. Fessler, and Douglas C. Noll \\ Corresponding Author: \\ Sydney N Williams \\ sydneynw@umich.edu \\ 734-647-1996 \\ Mailing Address: \\ Functional MRI Laboratory \\ 1072 BIRB \\ 2360 Bonisteel Blvd. \\ Ann Arbor, MI 48109-2108 \\ Word Count: 4824 \\ Institution: \\ Department of Biomedical Engineering \\ University of Michigan, Ann Arbor, MI, United States \\ Running Title: \\ Design of spectral-spatial phase prewinding pulses and their use in STFR steady-state imaging. \\ Keywords: \\ RF pulse design; field inhomogeneity; phase prewinding; spectral-spatial pulses
}

This is the author manuscript accepted for publication and has undergone full peer review but has not been through the copyediting, typesetting, pagination and proofreading process, which may lead to differences between this version and the Version record. Please cite this article as doi:10.1002/ mrm.26794. 


\begin{abstract}

\section{Purpose}

Spectrally selective "prewinding" radiofrequency pulses can counteract B0 inhomogeneity in steady-state sequences, but can only prephase a limited range of off-resonance. We propose spectral-spatial small-tip angle prewinding pulses that increase the off-resonance bandwidth that can be successfully prephased by incorporating spatially tailored excitation patterns.
\end{abstract}

\title{
Theory and Methods
}

We present a feasibility study to compare spectral and spectral-spatial prewinding pulses. These pulses add a prephasing term to the target magnetization pattern that aims to recover an assigned off-resonance bandwidth at TE. For spectral-spatial pulses, the design bandwidth is centered at the off-resonance frequency for each spatial location in a field map. We use these pulses in the small-tip fast recovery (STFR) steady-state sequence, which is similar to balanced steady-state free precession (bSSFP). We investigate improvement of spectral-spatial pulses over

spectral pulses using simulations and STFR scans of a gel phantom and human brain.

\section{Results}

In simulation, spectral-spatial pulses yielded lower normalized root mean squared excitation error than spectral pulses. For both experiments, the spectral-spatial pulse images are also qualitatively better (more uniform, less signal loss) than the spectral pulse images.

\section{Conclusion}

Spectral-spatial prewinding pulses can prephase over a larger range of off-resonance than their purely spectral counterparts.

Keywords:

$\mathrm{RF}$ pulse design; field inhomogeneity; phase prewinding; spectral-spatial pulses

\section{Introduction}

Inhomogeneity of the B0 field can cause spatially varying phase and signal loss in MRI. Spin-echo (SE) sequences can realign static off-resonance effects, but require a second refocusing RF pulse and may be unsuitable for short TR imaging or applications that are SAR limited (1). Assländer et al. introduced spectral prewinding pulses to compensate for spin dephasing in rapid gradient echo sequences (GRE), effectively combining the speed of GRE with the off-resonance robustness of SE (2), for a restricted off-resonance bandwidth.

Recently, spectrally selective phase prewinding pulses were incorporated into the small-tip fast recovery (STFR) steady-state sequence (3). The STFR sequence employs both a traditional "tipdown" pulse and then a "tip-up" pulse that quickly returns the magnetization to the longitudinal axis after free precession (4). STFR has mixed T2/T1 contrast and provides nearly equal signal 
level and tissue contrast in the human brain as balanced steady-state free precession (bSSFP). (5). One challenge with bSSFP is that banding artifacts may occur (6) that can only be removed with multiple acquisitions and phase cycling (7). With appropriately designed "tip-up" pulses, STFR can potentially eliminate the banding artifact in a single acquisition (4).

However, purely spectral prewinding pulses can refocus a limited range of frequencies (2). This paper proposes a spectral-spatial pulse that increases the effective prewinding bandwidth and mitigates the limitations of a purely spectral design. The key idea of this paper is to vary spatially the pulse design criterion based on voxel-by-voxel bulk off-resonance. The resulting pulse accommodates a wider effective bandwidth, improving signal recovery in inhomogeneous objects. Our proposed application for spectral-spatial STFR is brain imaging, where inhomogeneity in the B0 field is generally smoothly varying with a wide bandwidth.

In the following section we review spectral prewinding pulse design and introduce the extension to spectral-spatial prewinding pulses. We then formulate our constrained pulse design optimization problem under the small-tip angle (STA) approximation (8). Next, we outline pulse design validation experiments in a phantom and in vivo human brain. We quantify performance with subsequently defined metrics. Finally, we present simulation and experimental results for the phantom and in vivo pulse designs.

\section{Theory}

Spectral Prewinding Pulse Design

After RF excitation, the phase accumulation of spin isochromats that occurs during a free precession interval of length $\mathrm{T}_{\text {free }}$ is

$$
\theta_{\text {free }}=-2 \pi f \mathrm{~T}_{\text {free }}
$$

where $f$ denotes the off-resonance frequency (2). To compensate for this precession, a spectral design $(2,3)$ "prewinds" for phase by using the following target excitation pattern:

$$
d(f)=\alpha e^{2 \pi i \frac{\mathrm{T}_{\text {free }}}{2} f \cdot[2]}
$$

The spectral target pattern $d$ is a function of flip-angle $\alpha$ and off-resonance frequency $f$. The phase evolution is set to refocus at TE, which is half of $\mathrm{T}_{\text {free }}$ in STFR sequences. The target excitation pattern vector $\boldsymbol{d}=\left[d\left(f_{1}\right), . ., d\left(f_{N_{\mathrm{f}}}\right)\right]^{T}$ consists of samples of Eq. [2] at $N_{\mathrm{f}}$ frequencies over some bandwidth of interest.

Prewinding will be most effective if limited to one half of the unit circle, or a spread of less than or equal to $\pi$. In previous experiments, we have noticed this relationship as an empirical 
off-resonance bandwidth limit. Therefore we use

$$
\text { bandwidth } \leq \frac{1}{\mathrm{~T}_{\text {free }}}
$$

as a conseryative upper limit for prewinding. Eq. [3] suggests that the widest bandwidth (range of $f$ values used in Eq. [2]) for refocusing is obtained with a minimal free precession time. Free precession time is dictated by the data acquisition gradients which for certain applications and resolution might be too long. For example, a plausible $\mathrm{T}_{\text {free }}$ of $4 \mathrm{~ms}$ would conservatively allow for $\pm 125 \mathrm{~Hz}$ of refocusing and in some cases, this range is insufficiently wide to span the entire bandwidth of off-resonance frequencies at highly inhomogeneous regions within the human brain.

Spectral-Spatial Prewinding Pulse Design

To overcome the bandwidth limitations of the purely spectral prewinding design in (3), we propose a spectral-spatial RF pulse design approach that spatially adapts the spectral properties. The addition of spatial information allows the RF pulse to encode spatially varying off-resonance across the object. Even with a narrow design bandwidth, these pulses have the potential to track larger ranges of off-resonance by changing the design center frequency at each spatial location, provided these variations do not change too rapidly in space. Figure 1 illustrates this concept.

For the spectral approach, the target magnetization pattern $\boldsymbol{d}$ based on Eq. [2] for RF pulse design was simply a $1 \mathrm{D}$ vector corresponding to a range of off-resonance frequencies. In contrast, for the proposed spectral-spatial RF design approach, the target pattern is a function of two spatial dimensions and a spectral dimension. This feasibility study used a non-slice-selective 2D slice. For fully $3 \mathrm{D}$ imaging the spectral spatial pulse design would include three spatial dimensions and a $3 \mathrm{D}$ excitation trajectory. The target pattern $\boldsymbol{d}$ for spectral-spatial pulses consists of $N_{\mathrm{x}} \times N_{\mathrm{y}} \times N_{\mathrm{f}}$ samples of

$$
d(x, y, f)=\alpha e^{2 \pi i \frac{T_{\text {free }}}{2} f}
$$

where $N_{\mathrm{x}}$ and $N_{\mathrm{y}}$ are the number of samples in the $x$ and $y$ dimensions. The spatial aspect in this design is captured by the diagonal weighting matrix $\boldsymbol{W}$ (used in Eq. [6] below) that has diagonal elements corresponding to samples of the following $3 \mathrm{D}$ weighting function $w(x, y, f)$ :

$$
w(x, y, f)= \begin{cases}1, & |f-\Delta f(x, y)| \leq \frac{L}{2} \\ 0, & \text { otherwise }\end{cases}
$$

where $\Delta f(x, y)$ denotes the measured field map and $L$ is the user-defined local target bandwidth of the spectral-spatial pulse. In other words, $L$ defines the local bandwidth that the spectral- 
spatial pulse uses to encapsulate the spatially varying off-resonance as illustrated by the shaded red region in the bottom right of Figure 1. This combined spatial-spectral weighting is the unique characteristic of the proposed approach. If the weights were set to unity everywhere, then the spectral-spatial approach would revert to a pure spectral design. For additional exploration of local bandwidth $L$ and diagonal weighting matrix W please see Supporting Information Section A provided with this paper. Supporting Table S1 and Supporting Figure S1 explain the supplementary experiments; Supporting Table S2 and Supporting Figures S2-S3 present the results.

\section{Methods}

RF Pulse Design

Both purely spectral (2) and spectral-spatial pulses were designed using the small-tip angle approximation (8) for a target $2 \mathrm{D}$ slice. The STA approximation facilitates RF pulse design by establishing a linear Fourier relationship between RF waveform and transverse magnetization (9). The tip-down pulse design target pattern $\boldsymbol{d}$ was defined for spectral pulses by Eq. [2] and for spectral-spatial pulses by Eq. [4], where off-resonance frequencies $\boldsymbol{f}=\left[f_{1}, \ldots, f_{N_{\mathrm{f}}}\right]^{T}$ were sampled from the off-resonance range measured in a field map. The tip-up pulses used simulated magnetization magnitude and phase at the end of free precession to assign the magnitude and phase of the target tip-up pattern (3). The spectral-spatial pulse design used gradients played out during the $\mathrm{RF}$ pulse that trace out a variable density spiral k-space trajectory repeated multiple times. The speed and resolution of this trajectory permitted time between spirals to be spent at k-space center where we found that large amounts of RF energy are deposited.

We design the RF pulse by solving the STA constrained optimization problem:

$$
\begin{aligned}
& \hat{\boldsymbol{b}}=\underset{\boldsymbol{b}}{\operatorname{argmin}}\|\boldsymbol{A} \boldsymbol{b}-\boldsymbol{d}\|_{\boldsymbol{W}}^{2} \\
& \text { s.t. } C_{\mathrm{SAR}}\|\boldsymbol{b}\|_{2}^{2} \Delta t \leq p_{\max }
\end{aligned}
$$

where $\hat{\boldsymbol{b}}$ is the optimized RF pulse, $\boldsymbol{W}$ is the diagonal weighting matrix composed of samples of weighting function $w(x, y, f)$ (Eq. [5]), $C_{\mathrm{SAR}}$ is a conversion constant, and $\boldsymbol{A}$ is the small tip system matrix with elements $a_{j, n}=i \gamma M_{0} e^{2 \pi i\left(\boldsymbol{r}_{j} \cdot \boldsymbol{k}_{n}\right)} \Delta t$. Here, vector $\boldsymbol{r}_{j}=\left(x_{j}, y_{j}, f_{j}\right)$ contains spatial location samples $\left(x_{j}, y_{j}\right)$ and frequency samples $f_{j}$. Vector $\boldsymbol{k}_{n}$ contains excitation k-space samples $\left(k_{x, n}, k_{y, n}, k_{\mathrm{f}, n}\right)$. For prewinding pulses, $k_{\mathrm{f}, n}$ is simply the time-reversed vector for pulse length $\tau$ : $k_{\mathrm{f}, n}=t_{n}-\tau$. The matrix $\boldsymbol{A}$ is very large so we use Nonuniform Fast Fourier Transform (NUFFT) operations to perform matrix vector multiplications involving $\boldsymbol{A}(10)$.

Unlike traditional STA designs that penalize RF power with a regularization parameter (9), optimization problem Eq. [6] directly constrains the deposited RF power $p_{\max }$. We set the constraint $p_{\max }$ to a constant with units $\mathrm{W} / \mathrm{kg}$ chosen conservatively from SAR estimates from previous experiments using spectral pulses and the STFR sequence with the same TR. $C_{\mathrm{SAR}}$ is a measured 
constant that converts the integrated RF pulse $\|\mathbf{b}\|_{2}^{2} \Delta t$ from $\mathrm{G}^{2} \cdot \mathrm{ms}$ to $\mathrm{W} / \mathrm{kg}$ for a particular TR. We compute a 2D spectral-spatial pulse efficiently by minimizing Eq. [6] using approximately 100 iterations of the fast iterative shrinkage-thresholding algorithm (FISTA) (11).

Assuming a smoothly varying field map, we downsampled the $x$ and $y$ dimensions of the field map from $120 \times 120$ by a factor of 4 in forming $\left(x_{j}, y_{j}\right)$, and sampled off-resonance frequencies every $10 \mathrm{~Hz}$. Spatial downsampling has negligible effects on the RF excitation accuracy given the low resolution excitation k-space trajectories chosen for these pulse designs. The NUFFT matrix multiplication in Eq. [6] was implemented with the Michigan Image Reconstruction Toolbox, available for download at http://web.eecs.umich.edu/ fessler/.

Experiments

We performed the following experiments to examine if spectral-spatial prewinding pulses can recover larger off-resonance bandwidths than purely spectral pulses. This was evaluated via simulation and experimentally in a gel phantom and the human brain in vivo. We also simulated a non-prewinding hard pulse and compared to the spectral and spectral-spatial pulses in Supporting Information Section B provided with this paper. Supporting Table S3 and Supporting Figures S4-S6 show these results.

RF Pulse and Pulse Sequence

We performed all experiments with a birdcage single channel $\mathrm{T} / \mathrm{R}$ head coil on a $3 \mathrm{~T}$ GE MR750 scanner (GE Healthcare, Waukesha, WI). Using a field map from a 2D slice, we designed $3 \mathrm{~ms}$ tip-down and tip-up pulses for the STFR sequence (TE/TR=3.648 ms/14.08 ms), where TE is measured from the end of the RF pulse. For phantom experiments, we used a target flip angle of $\alpha=19.6^{\circ}$. For in vivo brain imaging we used target $\alpha=15.8^{\circ}$ based on previous literature findings showing optimal grey matter signal for STFR (4). For the gel phantom, we set $p_{\max }=2.2 \mathrm{~W} / \mathrm{kg}$ using $C_{\mathrm{SAR}}=525 \mathrm{~W} / \mathrm{kg} / \mathrm{G}^{2} / \mathrm{ms}$, and chose a stricter constraint of $p_{\max }=2.1 \mathrm{~W} / \mathrm{kg}$ using $C_{\mathrm{SAR}}=700$ $\mathrm{W} / \mathrm{kg} / \mathrm{G}^{2} / \mathrm{ms}$ for the in-vivo brain scan where we assumed increased RF coil loading. These are conservative power constraints below the SAR limitations within the head. The stopping criterion for RF pulse convergence in the FISTA algorithm was set so that the maximum difference between iterations normalized by the initialization pulse was less than $2^{-12}$ times the peak RF amplitude limit of 0.2 Gauss.

We designed the RF pulses with MATLAB (The MathWorks, Natick, MA). All pulses were designed online after the field map acquisition. The total time to estimate the object field map, design the RF pulses, and export the files to the scanner was less than 5 min. 


\section{Phantom Scans}

For phantom scans, we taped a small piece of metal from a paperclip to the surface of a homogenous gel ball phantom to induce B0 inhomogeneity. Two GRE scans (TE1/TE2/TR1/TR2/FA= $2.042 \mathrm{~ms} / 4.342 \mathrm{~ms} / 5.58 \mathrm{~ms} / 7.88 \mathrm{~ms} / 8.2^{\circ}$ ) with distinct echo times (TE1,TE2) were collected using a 3 D readout with $\mathrm{FOV}=240 \mathrm{~mm} \times 240 \mathrm{~mm} \times 296 \mathrm{~mm}$ and matrix size $120 \times 120 \times 74$. We used these two scans to generate a B0 field map that was estimated using the regularized method described in (12). We then chose a $2 \mathrm{D}$ slice with an off-resonance range of just over $\pm 100 \mathrm{~Hz}$.

For the phantom experiment, we designed two types of spectral pulses: one that was designed to cover the full bandwidth of off-resonance frequencies in the 2D field map, and another that centered the design at the mean off-resonance frequency and was restricted to the empirical bandwidth threshold from Eq. [3]. For a readout FOV $=240 \mathrm{~mm}$, matrix size 256, and $\pm 31.25 \mathrm{kHz} \mathrm{BW}$, the minimum free precession time was $\mathrm{T}_{\text {free }}=7.296 \mathrm{~ms}$ which gives a spectral prewinding bandwidth cutoff of $\pm 68.5 \mathrm{~Hz}$.

In preliminary simulations (not shown) we explored a variety of excitation k-space trajectories and compared their performance in simulation. We found that revisiting $\mathrm{k}$-space locations multiple times offers more complete coverage of k-space, albeit at lower spatial resolution, which is desirable for pulses with both spectral and spatial profiles. Based on these initial findings, we designed spectral-spatial pulses using two 2D variable density spiral (VDS) excitation k-space trajectories: one with three alternating spirals with $k_{x y \text {,max }}=0.13 \frac{\mathrm{cycles}}{\mathrm{cm}}$ and the other with two alternating spirals with $k_{x y \text {,max }}=0.20 \frac{\mathrm{cycles}}{\mathrm{cm}}$. For all spectral-spatial pulses we defined the weighting function $w(x, y, f)$ with $L=25 \mathrm{~Hz}$ (Eq. [5]). Therefore in total, two sets of spectral and two sets of spectral-spatial prewinding tip-down/tip-up pulses were designed for the phantom.

Experimental magnitude and phase images are presented using the STFR sequence. Phase images are computed by subtracting the inherent coil phase estimated from the TE1 GRE scan used for field map acquisition.

Human Scans

We also designed spectral and spectral-spatial pulses for a human volunteer's brain. Again, the first step was to obtain a field map with two GRE scans (using the same TE and TR values used in the phantom experiment) and select for pulse design a $2 \mathrm{D}$ slice with a significant range of off-resonance. Here, pulse design characteristics (spectral design bandwidth and excitation trajectory) were selected from the best spectral and best spectral-spatial pulses used in the phantom experiment, based on their quantitative performance metrics defined in the section below. The best spectral pulse was the pulse designed over the full off-resonance bandwidth, and the best spectral-spatial pulse used a VDS trajectory with two alternating spirals and $L=25 \mathrm{~Hz}$. Just two pulses were designed to limit scan time for the human volunteer. We also compare the spectral and spectral-spatial pulses to a simulated non-prewinding "hard" pulse with the same design flip angle 
$\alpha=15.8^{\circ}$ and $\mathrm{TE}=3.648 \mathrm{~ms}$.

Performance Metrics

Quantifying the excitation performance of the spectral-spatial RF pulses is nontrivial because we aim to match a particular phase and magnitude pattern across both spatial and spectral dimensions. Nevertheless the definitions of these metrics are critical for comparing various prewinding pulses. We measured the performance by simulating the magnetization for each voxel within our region of interest (ROI) at each frequency within the design bandwidth at that location. Magnetization values were computed in two ways: using STA approximation of the magnetization $\boldsymbol{m}_{\mathrm{STA}} \approx \boldsymbol{A} \boldsymbol{b}$

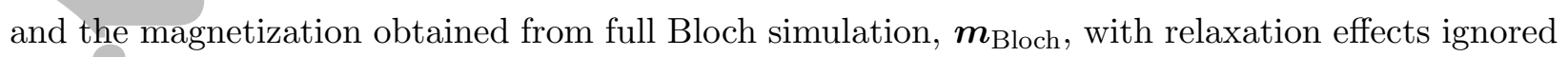
(T1 and T2 set to infinity). Our Bloch simulation results agreed with the STA approximation, so we report only the Bloch simulation results here. We conducted the multidimensional Bloch simulation on a voxel-by-voxel basis, accounting for within-voxel spin dephasing. We used a Gaussian model of the intravoxel frequency distribution (13) with a standard deviation of $\sigma=25 \mathrm{~Hz}$ (to reflect mean literature $\mathrm{R}^{*}$ values $\left.(14),(15)\right)$ and a simulation spread of $\pm 3 \sigma$. We evaluated the magnetization at the echo time TE.

At TE, ideally all spins within each voxel should rephase and produce uniform excitation at the target flip angle $\alpha$. To quantify excitation accuracy we calculated the Excitation Normalized Root Mean Squared Error (NRMSE)

$$
\text { Excitation NRMSE }=\frac{\sqrt{\frac{1}{N_{\mathrm{xy}}} \sum_{j=1}^{N_{x y}}\left|\sum_{n=1}^{N_{\mathrm{g}}} g_{n} m_{n, j}-\sin (\alpha)\right|^{2}}}{\sin (\alpha)} .
$$

Here the length $N_{\mathrm{g}}$ vector $\boldsymbol{g}$ contains the weights defined by the Gaussian model of intravoxel frequency distribution around the resonance frequency at each voxel; $\boldsymbol{g}$ is normalized to sum to 1. We compare Bloch simulation magnetization to the unitless target magnitude $\sin (\alpha)$. Under the STA approximation, $\sin (\alpha)$ is roughly equal to $\alpha$.

Another quality metric for the pulse is transverse magnetization uniformity, or how well offresonance spins within the spectral bandwidth come to the same mean magnetization. Using the simulated magnetization, we computed the mean magnitude across frequencies for each voxel, and finally defined the total mean and percent deviation of the magnetization magnitude as

$$
\bar{m}=\frac{1}{N_{\mathrm{xy}}} \sum_{j=1}^{N_{\mathrm{xy}}}\left(\sum_{n=1}^{N_{\mathrm{g}}} g_{n}\left|m_{n, j}\right|\right)
$$


and

$$
\% \sigma=\frac{\sqrt{\left.\frac{1}{N_{\mathrm{xy}}} \sum_{j=1}^{N_{\mathrm{xy}}}\left(\sum_{n=1}^{N_{\mathrm{g}}} g_{n}\left|m_{n, j}\right|\right)-\bar{m}\right)^{2}}}{\bar{m}} \times 100 \% .
$$

We also calculated the NRMSE of the excitation magnitude compared to the target transverse magnetization:

$$
\text { Magnitude NRMSE }=\frac{\sqrt{\frac{1}{N_{\mathrm{xy}}} \sum_{j=1}^{N_{\mathrm{xy}}}\left(\left|\sum_{n=1}^{N_{\mathrm{g}}} g_{n} m_{n, j}\right|-\sin (\alpha)\right)^{2}}}{\sin (\alpha)}
$$

Finally, we also calculate the phase error to evaluate the effectiveness of "prewinding". The desired phase of the magnetization will be zero at the echo time. The phase RMSE is

$$
\text { Phase RMSE }=\sqrt{\frac{1}{N_{\mathrm{xy}}} \sum_{j=1}^{N_{\mathrm{xy}}}\left(\angle \sum_{n=1}^{N_{\mathrm{g}}} g_{n} m_{n, j}\right)^{2}} .
$$

In conjunction, these various performance metrics provide insight into prewinding pulse performance in simulation, identifying how well pulses perform and under what conditions they might fail.

\section{Results}

Phantom Scans

Figure 2 shows the experimental field map used for spectral and spectral-spatial RF pulse design for the phantom scans. This figure also shows designed tip-down RF pulse and gradient waveforms for the two spectral (full bandwidth, theoretical bandwidth) and two spectral-spatial (3 spirals, 2 spirals) pulses. The theoretical spectral bandwidth limit is set to $\pm 68.5 \mathrm{~Hz}$ by the empirical relationship described in Eq. [3] while the entire range of off-resonance in this slice is $\approx 218 \mathrm{~Hz}$. All RF pulses designed were limited by the power constraint set in the optimization problem. By simulating on-resonance spins, this led to a median simulated flip angle of $\alpha=14.8^{\circ}$ and $15.3^{\circ}$ for the spectral pulses with full and theoretical bandwidths, respectively, and $\alpha=17.1^{\circ}$ and $17.9^{\circ}$ for the spectral-spatial pulses with 3 and 2 spirals respectively (compared to the target value of $19.6^{\circ}$ ).

Figure 3 shows the Bloch simulated magnetization in the phantom normalized by the target magnitude. Qualitatively, the simulations show a uniform magnetization magnitude slightly below 1. The RF pulses cannot exactly meet the nominal flip angle due to the active power constraint. In these simulations we also see roughly uniform, zero magnetization phase except for areas of 
high off-resonance by the paper clip. The phase from the purely spectral pulse designed over the theoretical bandwidth (Fig. 3a) performs particularly poorly, while the other spectral pulse and both spectral-spatial pulses have closer-to-zero phase.

Table 1 reports the performance metrics under Bloch simulation for these four designs. The spectral-spatial pulses outperform the purely spectral pulses overall. Spectral pulses suffer from large phase errors seen in the excitation NRMSE (0.81 and 0.66) and phase RMSE (33.3 ${ }^{\circ}$ and $\left.28.5^{\circ}\right)$. The spectral-spatial pulses have lower performance metric error while still maintaining high mean magnitude, especially for the spectral-spatial pulse using a two-spiral variable density spiral excitation trajectory. This pulse produces the lowest error in every case.

Figure 4 shows the experimental magnitude and phase images of the phantom for the four RF pulses. Just like in simulation, these results demonstrate the improved quality of spectral-spatial pulses compared to spectral pulses. The spectral-spatial magnitude images exhibit less signal loss than the purely spectral magnitude images and the phase images are closer to zero for spectralspatial pulses, indicating more accurate prephasing.

Human Scans

The field map for the human brain slice used for pulse design had an off-resonance range of about $\pm 85 \mathrm{~Hz}$ (Fig. 5). Figure 5 also shows the k-space of phase induced by off-resonance with overlay of excitation k-space trajectory, the RF and gradient waveforms for the spectral and spectral-spatial pulses designed for this slice, and simulated relative complex error $\left(\left|\frac{m_{\mathrm{xy}}}{\sin \alpha}-1\right|\right)$ for the spectral-spatial pulse as a function of one spatial dimension and all frequencies. In this case just one spectral and one spectral-spatial pulse were designed. Again, the RF pulses were limited by the power constraint, and the median simulated on-resonance flip angle was $\alpha=8.6^{\circ}$ and $14.9^{\circ}$ for the spectral and spectral-spatial pulses, respectively. Figure $5 \mathrm{~b}$ was computed as the 2D Fourier Transform of $\exp (-2 \pi i \mathrm{TE} \Delta f(x, y))$. In this subfigure, we see that the 2VDS excitation captures the majority of the field map energy predominantly at the k-space center.

Figure 6 displays the Bloch simulated magnetization for the two prewinding RF pulse designs and Table 2 reports performance metrics. Again, the spectral-spatial pulse outperforms the purely spectral pulse for all metrics. Notably, the excitation NRMSE and phase RMSE drop from 0.54 and $25.2^{\circ}$ in the spectral case to 0.18 and $7.0^{\circ}$ in the spectral-spatial case, respectively.

Additional intuition about pulse performance can be gained by plotting error as a function of spatial location. Figure 7 shows excitation and phase root squared error combined across all simulated frequencies as a function of spatial location for the simulated hard pulse, purely spectral pulse, and spectral-spatial pulse. In this plot, we can see that the hard pulse performs poorly, the spectral pulse performs moderately well, and the spectral-spatial pulse performs best.

Figure 8 shows the in vivo experimental magnitude and phase images for the spectral and spectral-spatial RF pulse. The spectral-spatial pulse magnitude image has less signal intensity variation than the purely spectral pulse, while the spectral-spatial phase image is also closer to uni- 
formly zero. Therefore, qualitatively the spectral-spatial pulse has better off-resonance prewinding capability than the spectral pulse.

\section{Discussion}

The principal idea behind spectral-spatial pulses is that the combination of spectral prewinding with spatial encoding of off-resonance patterns using magnetic field gradients allows for improved signal rephasing compared to purely spectral pulses alone, as shown in Fig. 1. This is useful because purely spectral pulses are limited in the off-resonance bandwidth that can they can recover, which is related to sometimes non-negotiable pulse sequence parameters such as flip angle and echo time. We believe this new pulse has useful applications in the human brain, where off-resonance can vary smoothly but cover a large range (bandwidth). This paper demonstrates the utility of spectralspatial pulses using a phantom and in vivo brain example with the STFR sequence. The STFR sequence is useful for tailored RF pulses because the unique tip-up pulse helps accommodate any magnetization error between the target pattern and Bloch simulated magnetization of the tip-down pulse. This makes spectral-spatial prewinding pulses a good match to the STFR pulse sequence.

In the phantom experiment, Fig. 3 and Table 1 show dramatic qualitative and quantitative improyement when comparing spectral-spatial to purely spectral prewinding pulses. In particular, the excitation NRMSE, which captures the pulse's ability to rephase intravoxel spin isochromats with spectral prewinding, is roughly halved for both spectral-spatial excitation trajectory designs. Furthermore, the experimental images (Fig. 4) confirm the simulation results and the spectralspatial pulses create more uniform images for the same flip angle design. The presence of slight, darkened signal loss regions in the spectral-spatial phantom images might reflect the limited spatial resolution of the spectral-spatial excitation k-space trajectory.

The in vivo experiments exhibited similar results. Again, the spectral-spatial pulse outperformed the purely spectral pulse as highlighted in Fig. 8 and Table 2, with excitation NRMSE again reducing to half. There are significant visual differences between the in vivo images (Fig. 8). The spectral-spatial pulse produces a brighter, more uniform magnitude image. The spectral-spatial pulse magnitude image does not have notable areas of signal loss, in contrast to the darker rostral region in the spectral magnitude image. In addition to prewinding performance improvements, some of the brightness differences between these two pulses might also be attributed to the active power constraint in the RF pulse design which may inhibit the RF pulse from realizing the nominal flip angle. Both magnitude images exhibit bright cerebrospinal fluid (CSF) that is typical for the mixed T2/T1 contrast of steady-state sequences like STFR and bSSFP (4). Additionally, the in vivo phase images also illustrate the improved performance of spectral-spatial pulses. The phase at TE is noticeably more uniform in the spectral-spatial pulse phase image compared to the purely spectral phase image.

Despite their enhanced performance compared to purely spectral pulses, there are a few limitations to spectral-spatial pulse design. First, the excitation k-space trajectory is restricted by the 
length of the RF pulse and gradient amplitude and slew rate limits. This often means that the excitation trajectories do not extend very far into $k_{x y}$, resulting in low excitation resolution. In many cases, the off-resonance in the human brain varies slowly enough that with even low resolution the spectral-spatial pulse can encode the spatially varying off-resonance. However, in regions where the field map contains a steep off-resonance gradient, the spectral-spatial pulse will not fully capture this transition and may perform no better than a purely spectral pulse. Furthermore, when moving to $3 \mathrm{D}$ spectral-spatial designs, there will be further limitations in resolution, though this limitation can be eased by using parallel excitation.

Although this paper explored spiral trajectories to understand how the trajectory affects spectralspatial pulse performance, by no means was this search exhaustive. There is still a need to better understand the trade-off between $\mathrm{k}$-space extent in $k_{x y}$ and other pulse design parameters such as target bandwidth $L$. One possible way of addressing this problem is the joint design of optimal k-space trajectories and RF pulses. In recent work by Sun et al. (16) and Davids et al. (17) the joint design problem is considered for the application of inner-volume excitation. Joint optimization of the k-space trajectory/gradient waveform is a natural extension of our RF pulse design problem.

Another important factor for spectral-spatial pulse design is that it must occur online. For the results shown, the pulse design routine increased scan session duration minimally. Nevertheless it is still possible that patients will move between field map scan and acquisition with the designed pulse, compromising the spatially varying pulse design. Therefore reducing motion and time in the scanner is critical. For purely spectral pulses, motion is less problematic because the pulses are not spatially resolved.

The work presented here is a feasibility study that uses 3D readout for a pulse designed to a particular 2D slice. For this concept to become practically useful, we will need to expand our design to three spatial dimensions. One benefit of 3D spectral and spectral-spatial pulse designs is that they can help account for field map variation in the slice dimension, but the higher dimensionality could increase the online pulse design time significantly. An alternative experiment could be constructed for a slice-selective spectral-spatial prewinding pulse. This is challenging because a third gradient dimension, the slice-select gradient, is introduced. We imagine that "fast k-z" (20) or spokes (21) trajectories could be used. Here, we would have to face the competing needs of high $k_{z}$ extent for selecting relatively thin slices yet also producing enough spokes for moderate sampling in the $k_{x y}$ plane.

We designed the spectral-spatial pulses using the small-tip angle approximation. Even though the RF pulses may be designed for a certain low flip angle such as $\alpha=15.8^{\circ}$ as used in our human experiments, the instantaneous flip during the RF pulse may be higher. In (3), Sun et al. demonstrated that spectral pulses can exceed $90^{\circ}$ when the integrated power is relatively high (or Tikhonov regularization term is low). For spectral-spatial pulses, the presence of magnetic field gradients makes the constraining of instantaneous flip angles more difficult; the flip angle is no longer simply proportional to the integral of the RF pulse with time. However, the agreement we observe between the STA model and Bloch simulation suggests that the STA approximation 
was adequate for these designs, perhaps due in part to our integrated power constrained design. The need for the current RF pulse design approach to satisfy the STA approximation means that spectral and spectral-spatial pulse designs should be monitored for flip angle violations, especially when the target flip angle is high.

Here we designed spectral and spectral-spatial prewinding pulses with fixed constraints on integrated power and found that spectral-spatial pulses performed with lower error for all quantitative metrics. Conversely, we would expect that designing spectral and spectral-spatial pulses for the same error values would result in the spectral-spatial pulses having lower integrated power. This formulation might have useful applications when SAR deposition is the primary concern. Furthermore, we have only reported the equivalent SAR constraints from our RF pulse optimization as reported on the scanner during imaging. In the future, we would like to have an efficient method for estimating SAR deposition during the design of RF pulses so that the constraints are applied directly in terms of $\mathrm{W} / \mathrm{kg}$.

Tailored RF pulses such as the spectral-spatial pulses explored in this paper depend on the accuracy of the magnetic field gradients. Distortion and timing errors between gradients and RF pulse is a common problem that can degrade excitation accuracy (18). In our experiments, we pre-measured gradients using the method in (19) and did not notice significant deviation between prescribed and measured excitation trajectories. However, as we design further spectral-spatial pulses with other excitation trajectories it may become necessary to evaluate gradient errors and perhaps adopt novel correction schemes such as that described in Harkins et al. (18).

In the future, we plan to extend our spectral-spatial pulse design to three spatial dimensions. Another plan is to tailor the pulse design to the STFR sequence by adjusting the design for its performance in the steady-state (whereas tip-down/tip-up pairs are currently designed for one cycle of STFR). Finally, we will examine a number of clinical imaging applications such as optic nerve, lung, or bowel imaging where these prewinding pulses might be particularly useful.

\section{Conclusions}

This paper has introduced a spectral-spatial phase prewinding RF pulse that extends the spectral pulses introduced by Assländer et al. (2). These pulses can compensate for field inhomogeneity induced phase by prewinding the phase such that, after excitation, spins refocus at the echo time. While purely spectral pulses are limited to a modest off-resonance bandwidth, introducing excitation k-space provides spatially varying off-resonance coverage. This approach can create a wider effective recovery bandwidth that reduces signal loss. We demonstrated this concept by designing spectralspatial pulses with variable density spiral k-space trajectories. We compared spectral-spatial pulses to their purely spectral counterparts with simulation, phantom studies, and in vivo human brain studies using the STFR steady-state sequence. The results confirm that spectral-spatial pulses do in fact improve recovery of signal loss due to off-resonance when compared to purely spectral prewinding pulses. 


\section{References}

[1] Hargreaves B. Rapid gradient-echo imaging. J Magn Res Im 2012;36:1300-1313.

[2] Assländer J, Glaser SJ, Hennig J. Spin echoes in the regime of weak dephasing. Magn Res Med 2016;75:150-160.

[3] Sun H, Fessler JA, Noll DC, Nielsen JF. Balanced SSFP-like steady-state imaging using smalltip fast recovery (STFR) sequence with a spectral pre-winding pulse. Magn Res Med 2016; $75: 839-844$.

[4] Nielsen JF, Yoon D, Noll DC. Small-tip fast recovery imaging using non-slice-selective tailored tip-up pulses and radiofrequency-spoiling. Magn Res Med 2013;69:657-666.

[5] Sun H, Fessler JA, Noll DC, Nielsen JF. Strategies for improved 3D small-tip fast recovery (STFR) imaging. Magn Res Med 2014;72:389-98.

[6] Bieri O, Scheffler K. SSFP signal with finite RF pulses. Magn Res Med 2009;62:1232-41.

[7] Çukur T, Shimakawa A, Yu HY, Hargreaves BA, Hu BS, Nishimura DG, Brittain JH. Magnetization-prepared ideal bSSFP: a flow-independent technique for noncontrast-enhanced peripheral angiography. J Magn Res Im 2011;33:931-939.

[8] Pauly J, Nishimura D, Macovski A. A k-space analysis of small-tip-angle excitation. J Magn Res 1989;81:43-56.

[9] Yip C, Fessler JA, Noll DC. Iterative RF pulse design for multidimensional, small-tip-angle selective excitation. Magn Res Med 2005;54:908-17.

[10] Fessler JA, Sutton BP. Nonuniform fast Fourier transforms using min-max interpolation. IEEE Trans Sig Proc 2003;51:560-74.

[11] Beck A, Teboulle M. A fast iterative shrinkage-thresholding algorithm for linear inverse problems. SIAM J Imaging Sci 2009;2:183-202.

[12] Allison MJ, Fessler JA. Accelerated computation of regularized field map estimates. In Proc Intl Soč Magn Reson Med, Melbourne May 5-11, 2012. p. 0413.

[13] Mulkern RV, Balasubramanian M, Mitsouras D. On the Lorentzian versus Gaussian character of time-domain spin-echo signals from the brain as sampled by means of gradient-echoes: implications for quantitative transverse relaxation studies. Magn Res Med 2015;74:51-62.

[14] Olafsson VT, Noll DC, Fessler JA. Fast joint reconstruction of dynamic $R_{2}^{*}$ and field maps in functional MRI. IEEE Trans Med Imag 2008;27:1177-88.

[15] Dahnke H, Schaeffter T. Limits of detection of spio at 3.0T using $T_{2}^{*}$ relaxometry. Magn Res Med 2005;53:1202-1206. 
[16] Sun H, Fessler JA, Noll DC, Nielsen JF. Rapid inner-volume imaging in the steady-state with 3D selective excitation and small-tip fast recovery (STFR) imaging. Magn Res Med 2016; $76: 1217-1223$.

[17] Davids M, Schad LR, Wald LL, B. Fast three-dimensional inner volume excitations using parallel transmission and optimized k-space trajectories. Magn Res Med 2016;76:1170-1182.

[18] Harkins KD, Does MD, Grissom WA. Iterative method for predistortion of MRI gradient waveforms. IEEE Trans Med Imag 2014;33:1641-1647.

[19] Duyn JH, Yang Y, Frank JA, van der Veen JW. Simple correction method for k-space trajectory deviations in MRI. J Magn Res 1998;132:150-3.

[20] Saekho S, Yip C, Noll DC, Boada F, Stenger VA. Fast-kz three-dimensional tailored radiofrequency pulse for reduced B1 inhomogeneity Magn Res Med 2006;55:719-24.

[21] Zelinski A, Wald LL, Setsompop K, Goyal V, and Adalsteinsson E. Sparsity-enforced sliceselective MRI RF excitation pulse design. Magn Res Med 2008;27:1213-29.

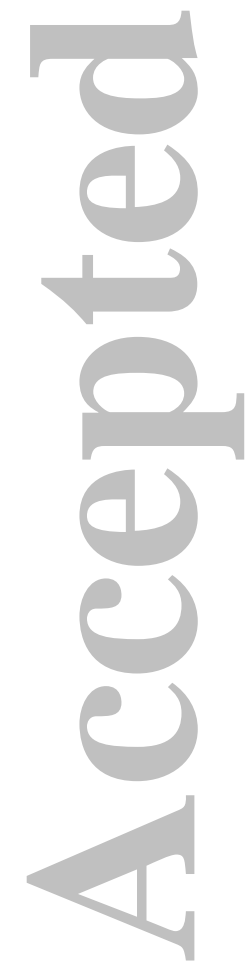




\section{Figure Captions}
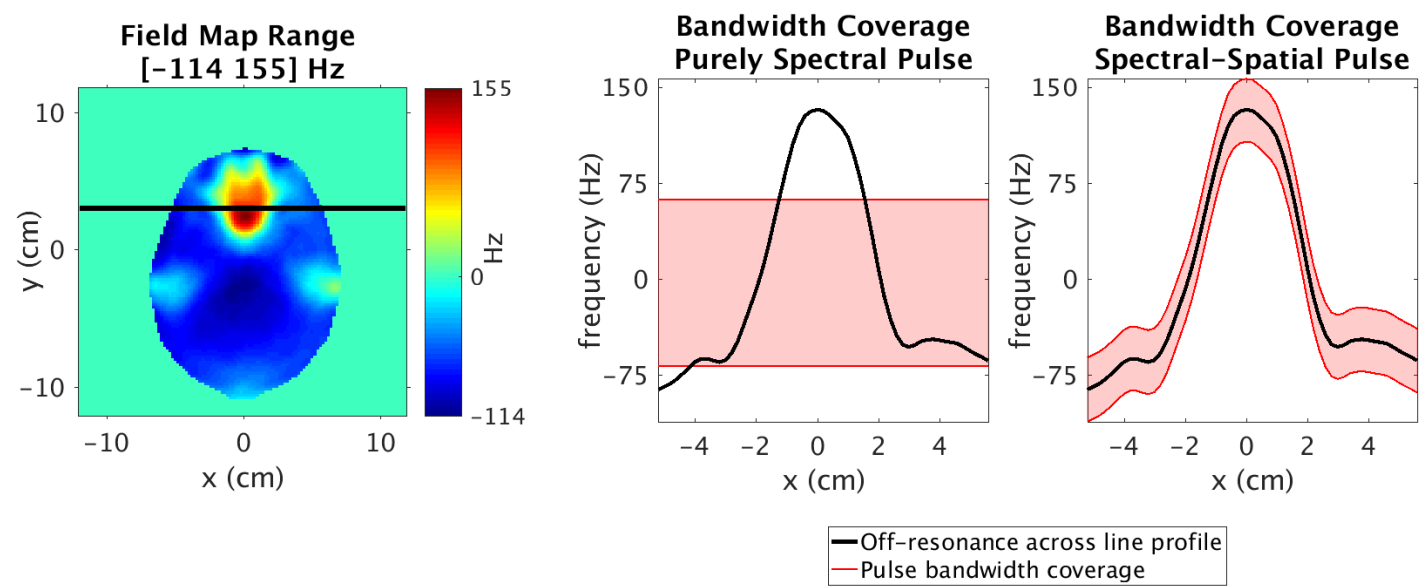

Figure 1: 1D conceptual representation of purely spectral and spectral-spatial prewinding pulse design. The left figure shows a typical human brain field map at a slice taken slightly above the sinuses, containing a significant range of off-resonance. A 1D line profile across one y-location of this field map is plotted in black on the right figures. The purely spectral pulse (center) does not vary the target recovery bandwidth spatially, while the spectral-spatial pulse (right) does. 


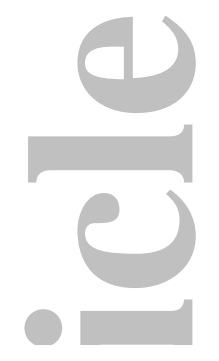

Field Map

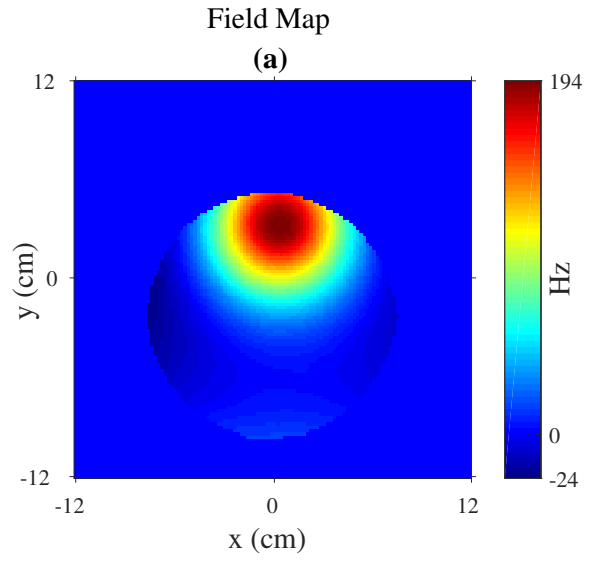

Tip-down RF Pulses and Gradient Waveforms

(b)
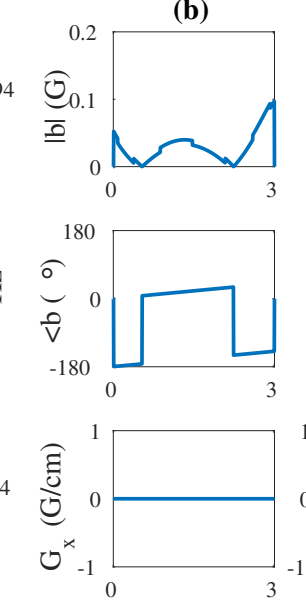
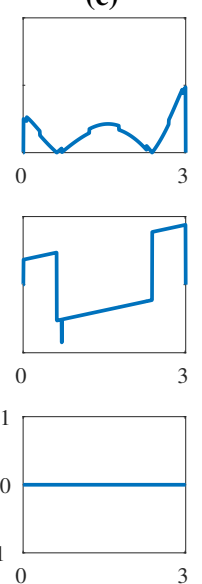

(d)
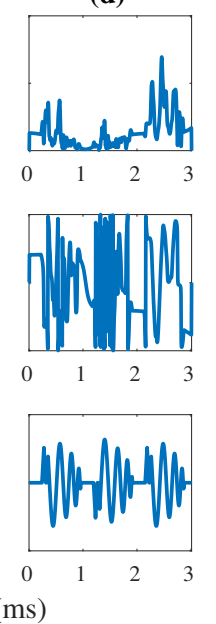

(e)
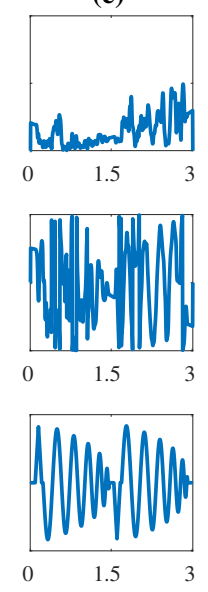

Figure 2: (a) Ball phantom field map used to design spectral and spectral-spatial prewinding pulses. (b-e) Tip-down RF pulse magnitude, phase, and excitation gradients for spectral pulses with (b) $\pm 68.5 \mathrm{~Hz}$ target bandwidth and (c) full field map off-resonance bandwidth, and 2D spectral-spatial pulses with (d) 3VDS excitation trajectory and (e) 2VDS excitation trajectory. Only the $G_{x}$ waveform is shown. Further description of these VDS trajectories is found in the Methods section. 

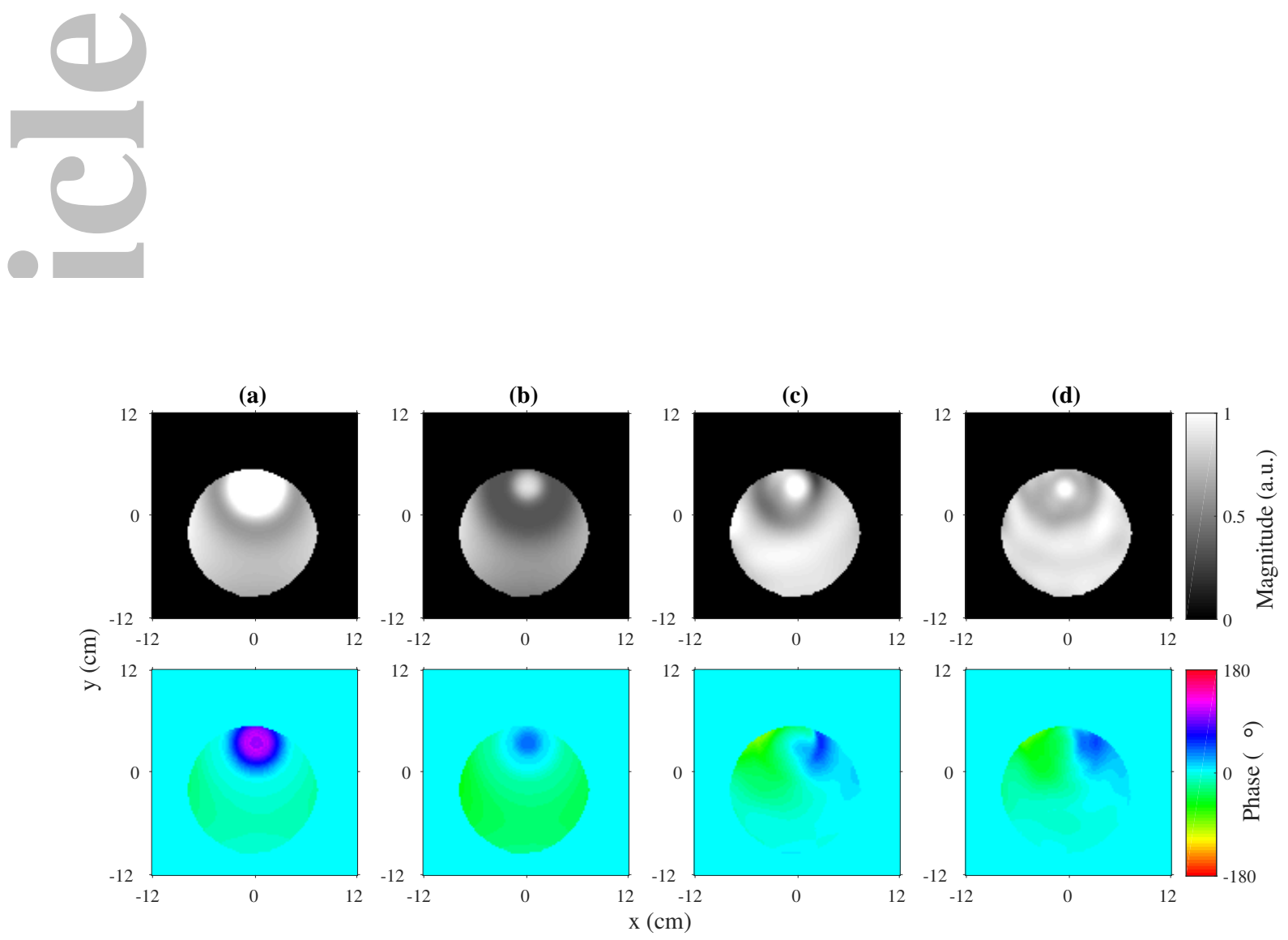

Figure 3: Bloch simulated magnetization magnitude (top row) and phase (bottom row) at TE for spectral pulses with (a) $\pm 68.5 \mathrm{~Hz}$ target bandwidth and (b) full field map off-resonance bandwidth, and 2D spectral-spatial pulses with (c) 3 variable density spiral (VDS) excitation trajectory and (d) 2VDS excitation trajectory. The magnitude images are more uniform and the phase images are closer to zero in the spectral-spatial simulations $(\mathrm{c}, \mathrm{d})$. 

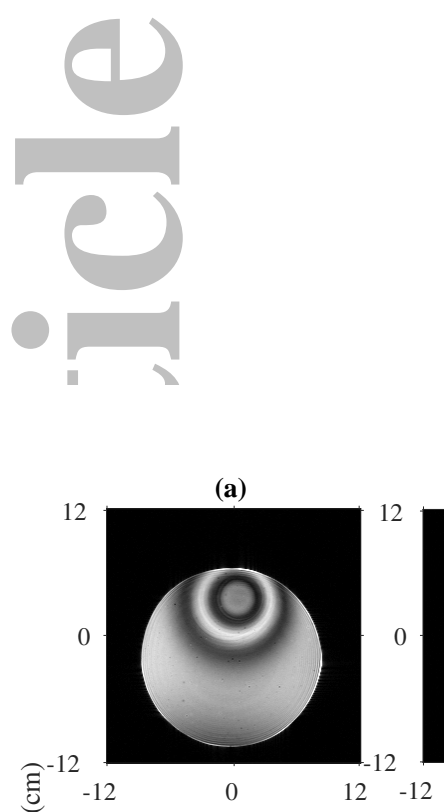

(b)

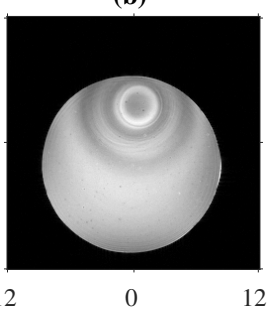

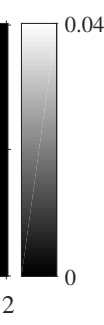

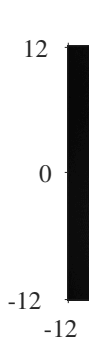

(c)
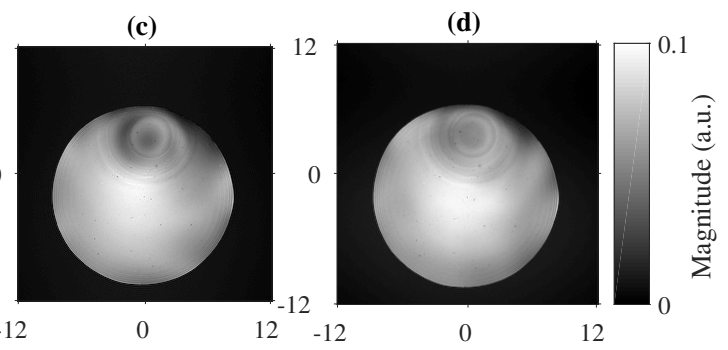

12
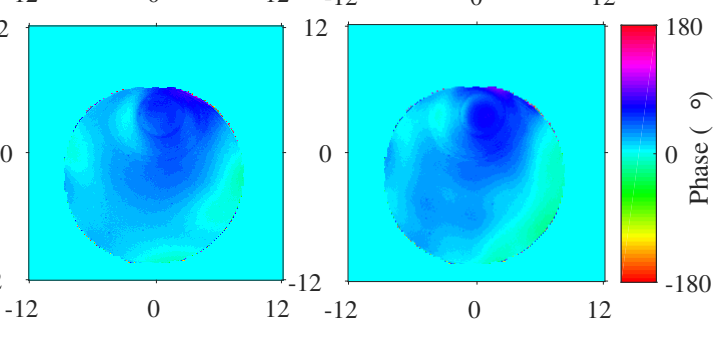

Figure 4: Experimental magnitude (top row) and phase (bottom row) STFR images for spectral pulses with (a) $\pm 68.5 \mathrm{~Hz}$ target bandwidth and (b) full field map off-resonance bandwidth, and 2D spectral-spatial pulses with (c) 3VDS excitation trajectory and (d) 2VDS excitation trajectory. Magnitude images are scaled differently for the spectral images and the spectral-spatial images to facilitate visual comparison. As in the simulation, the magnitude images are more uniform and the phase images are closer to zero for spectral-spatial pulses.

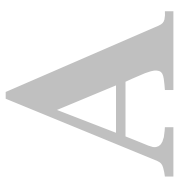



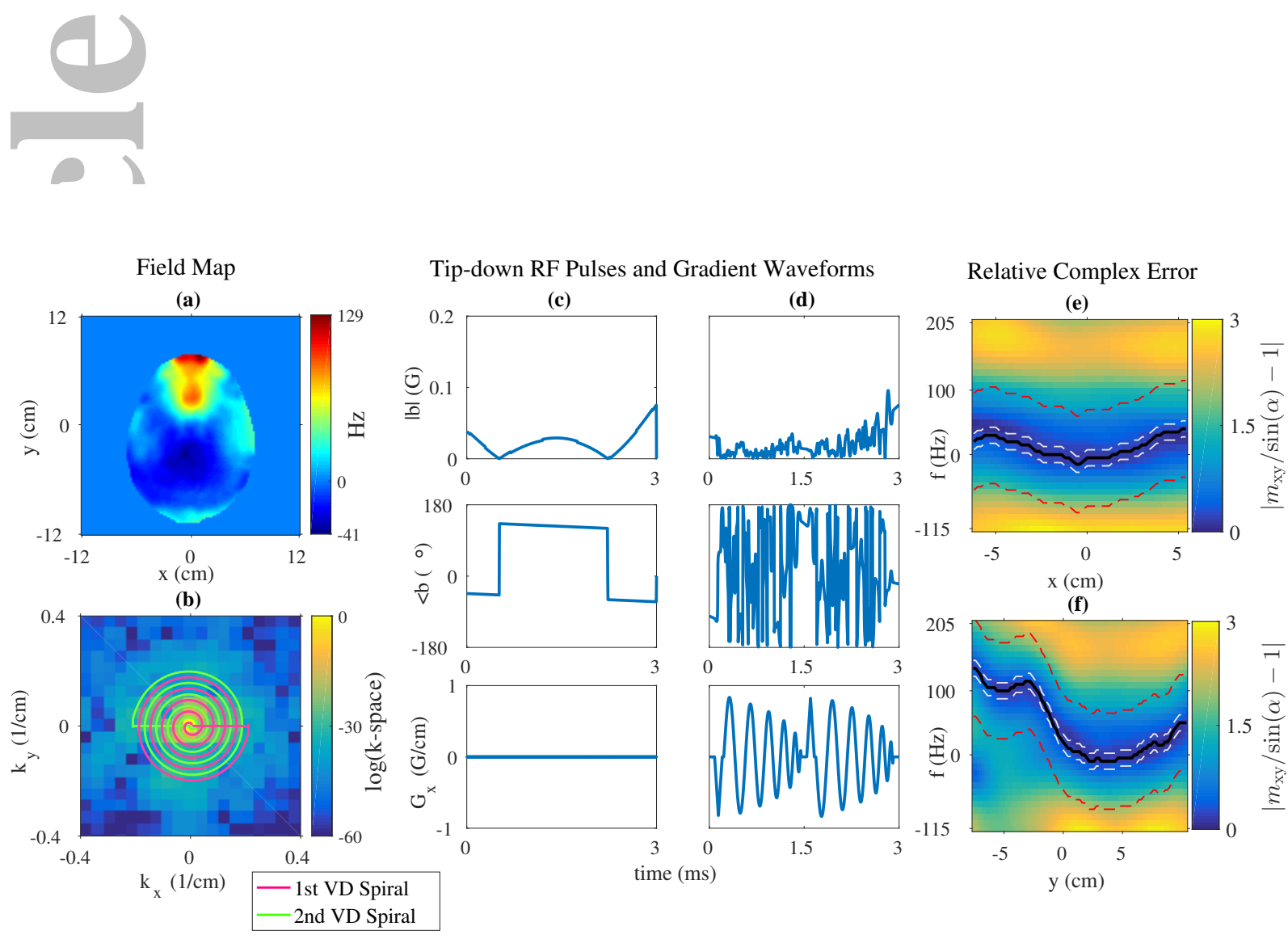

Figure 5: (a) Human brain field map used to design spectral and spectral-spatial prewinding pulses and (b) logarithm of zoomed k-space of phase induced by off-resonance with overlay of excitation k-space trajectory, 2VDS. (c-d) Tip-down RF pulse magnitude, phase, and $G_{x}$ excitation gradients for (c) a spectral pulse designed with full field map off-resonance bandwidth and (d) a 2D spectralspatial pulse designed with the same 2VDS excitation trajectory used in the phantom experiments. (e-f) Relative complex error $\left|\frac{m_{\mathrm{xy}}}{\sin \alpha}-1\right|$ for the spectral-spatial pulse as a function of frequency and 1D line profile across (e) all $x$ at $y=2.8 \mathrm{~cm}$ and across (f) all $y$ at $x=0.2 \mathrm{~cm}$. The dashed white lines represent the $L=25 \mathrm{~Hz}$ used as the local bandwidth for the spectral-spatial pulse, and the dashed red lines represent the $\pm 3 \sigma=75 \mathrm{~Hz}$ bandwidth used in Bloch simulation. Further examples of these plots are found in Supporting Info B. 


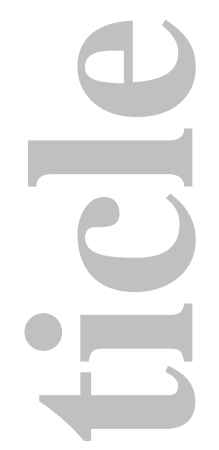

(a)

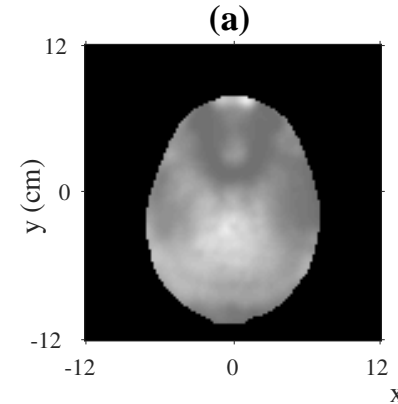

$\mathrm{x}(\mathrm{cm})$

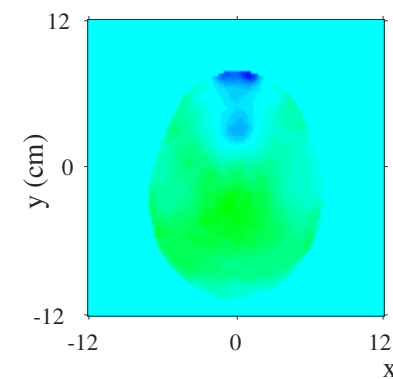

(b)
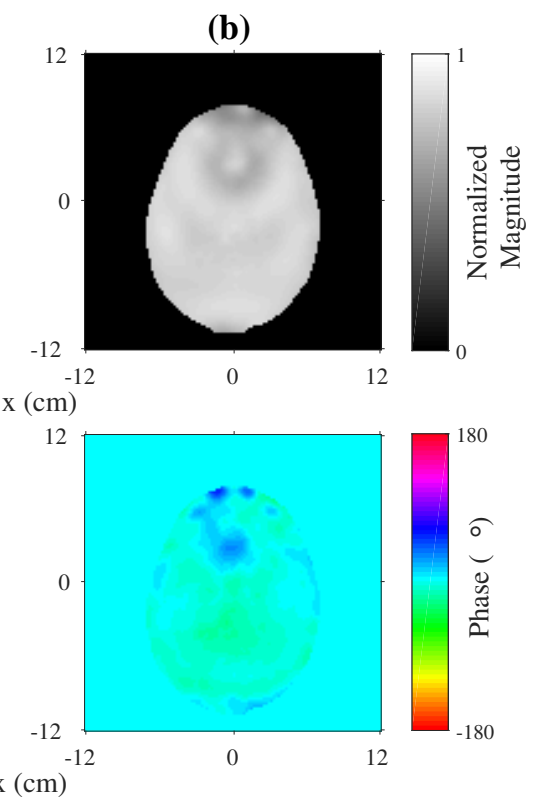

Figure 6: Bloch simulated magnitude (top row) and phase (bottom row) at TE for (a) a purely spectral tipdown pulse and (b) a spectral-spatial tipdown pulse designed for the 2D field map in Fig. 5(a). The spectral-spatial pulse suffers from less signal loss and obtains a flatter phase closer to zero. 
(a)

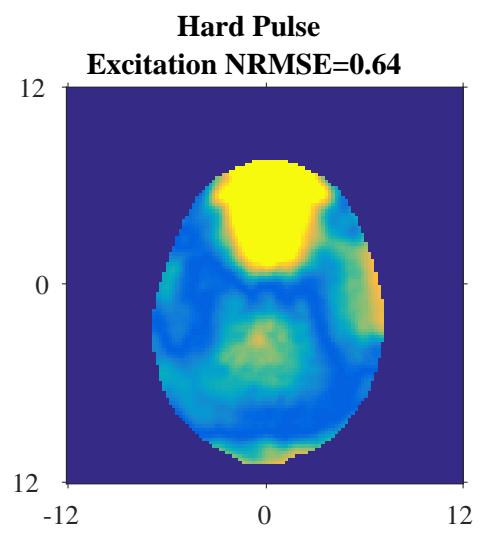

Hard Pulse

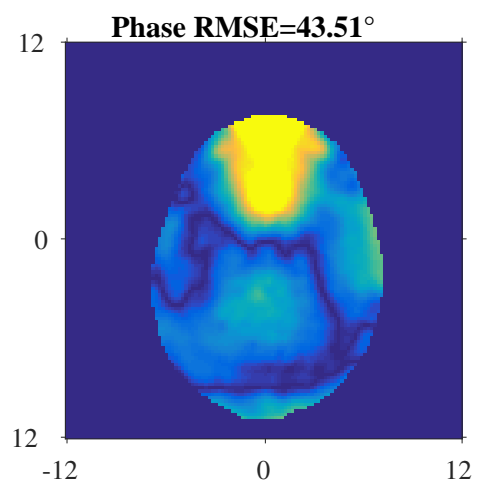

(b)

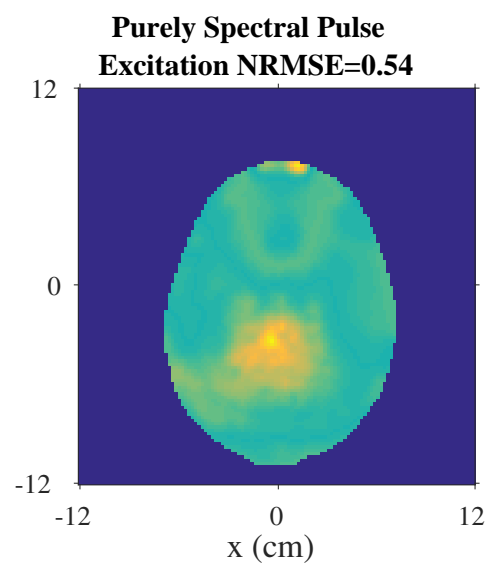

Purely Spectral Pulse

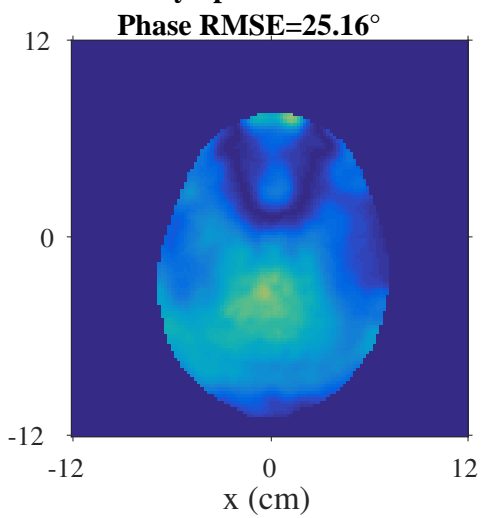

(c)

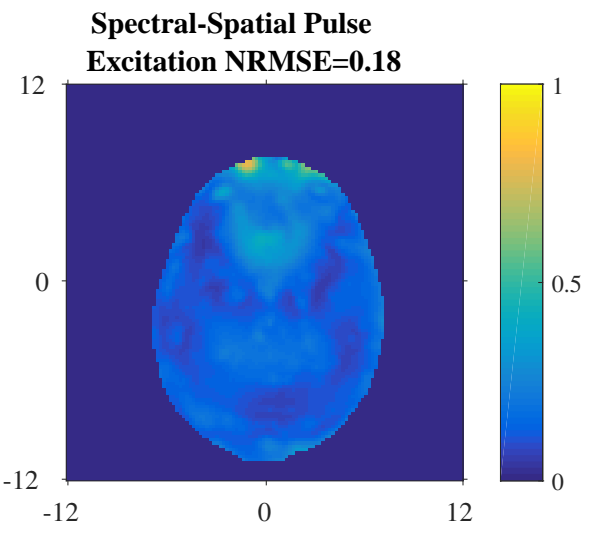

Spectral-Spatial Pulse

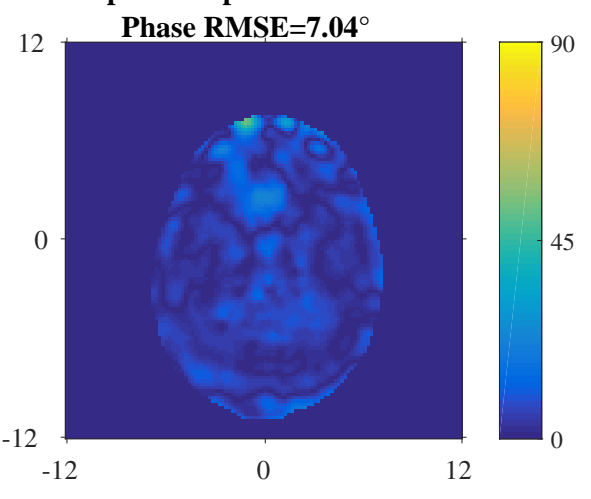

Figure 7: Simulated error as a function of space. Excitation root squared error (top row) and phase root squared error (bottom row) across a range of intravoxel frequencies (Gaussian distribution over $[-3 \sigma: 3 \sigma]$ with $\sigma=25 \mathrm{~Hz}$ ) over all spatial locations $x, y$ for (a) the hard pulse, (b) the purely spectral pulse, and (c) the spectral-spatial pulse. The excitation and phase errors are lowest in the spectral-spatial case, and the same is true for the overall excitation NRMSE and phase RMSE. 


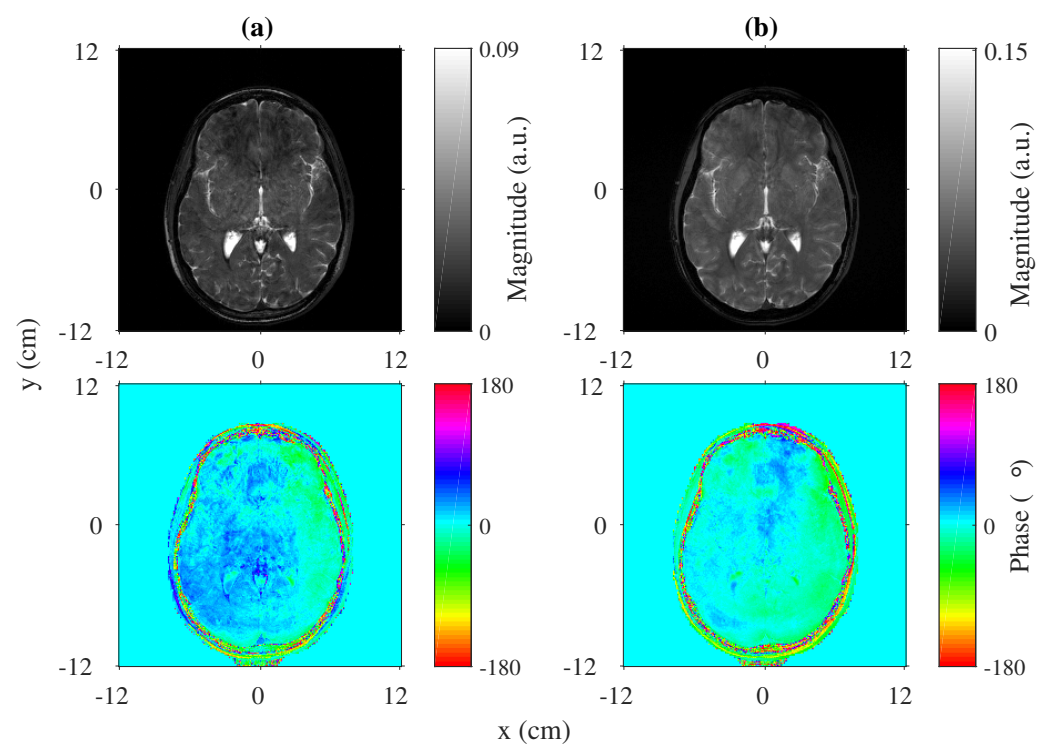

Figure 8: Experimental magnitude (top row) and phase (bottom row) images for (a) a spectral pulse designed over full field map off-resonance bandwidth and (b) a 2D spectral-spatial pulse designed with 2VDS excitation trajectory. Again, magnitude images are scaled differently for the spectral images and the spectral-spatial images to help visual comparison. The spectral-spatial pulse yields more uniform magnitude and closer to zero phase.

Suppōrting Figure S1: Four possible design weighting matrices $\mathbf{W}$ for spectral-spatial pulse prewinding. In the top row, the intravoxel bandwidth spread is universally $L_{\text {total }}(x, y)=25 \mathrm{~Hz}$ at all locations. In the bottom this value varies spatially with the spread proportional to throughplane gradient. In the left column, the values included in $\mathbf{W}$ are binary (0's or 1's). In the right column, the values are weighted by a normalized Gaussian distribution. In the main paper, we used the top left design weighting matrix.

Supporting Figure S2: Simulated magnitude images for all possible design weighting matrix and simulation combinations presented in Table S1.

Supporting Figure S3: Simulated phase images for all possible design weighting matrix and simulation combinations presented in Table S1.

Supporting Figure S4: Hard pulse simulation results. Top row: 2D field map (left), simulated magnitude (center), and simulated phase (right). Middle row: relative complex error, relative magnitude error, and absolute phase error for the 1D line profile magnitude across all $x$ at $y=2.8$ $\mathrm{cm}$. Bottom row: relative complex error, relative magnitude error, and absolute phase error for profile across all $y$ at $y=0.2 \mathrm{~cm}$. The dashed white lines represent the $L=25 \mathrm{~Hz}$ used as the local bandwidth for the spectral spatial pulse, and the dashed red lines represent the $\pm 3 \sigma=75 \mathrm{~Hz}$ bandwidth used in Bloch simulation. These plots are repeated for the spectral and spectral-spatial pulses in Supp. Figs S5 and S6. 


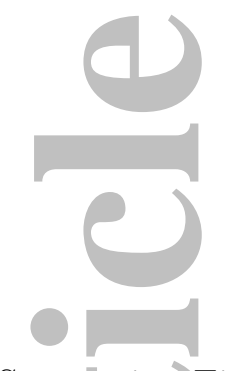

Supporting Figure S5: Purely spectral pulse simulation results. Compare with Supp. Figs S4 and S6.
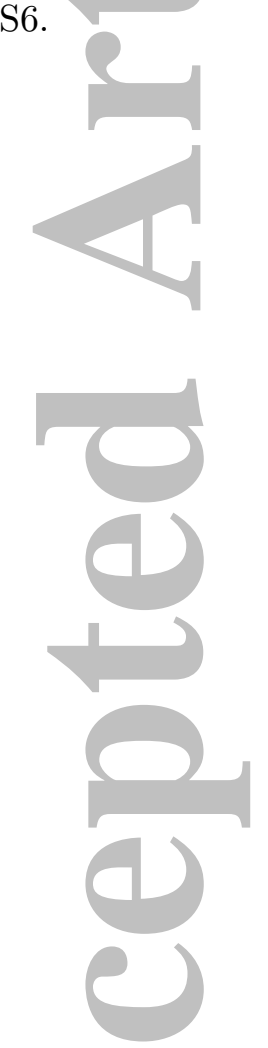

Supporting Figure S6: Spectral-spatial pulse simulation results. Compare with Supp. Figs S4 and S5.

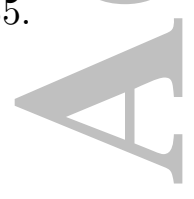




\section{Tables}

\begin{tabular}{|l|l|l|l|l|l|}
\hline $\begin{array}{l}\text { Pulse Design } \\
\text { Trajectory }\end{array}$ & $\begin{array}{l}\text { Excitation } \\
\text { NRMSE }\end{array}$ & $\begin{array}{l}\text { Phase } \\
\text { RMSE }\left(^{\circ}\right)\end{array}$ & Mean Mag. & \% St. Dev. & $\begin{array}{l}\text { Magnitude } \\
\text { NRMSE }\end{array}$ \\
\hline $\begin{array}{l}\text { Spectral } \\
\text { BW-limited, } \\
\pm 68.5 ~ H z\end{array}$ & 0.81 & 33.3 & $\mathbf{0 . 3 0}$ & 36.6 & 0.34 \\
\hline $\begin{array}{l}\text { Spectral } \\
\text { full BW }\end{array}$ & 0.66 & 28.5 & 0.18 & 26.6 & 0.48 \\
\hline $\begin{array}{l}\text { Spectral- } \\
\text { spatial 3 VDS }\end{array}$ & 0.41 & 23.4 & 0.28 & 18.9 & 0.24 \\
\hline $\begin{array}{l}\text { Spectral- } \\
\text { spatial 2 VDS }\end{array}$ & $\mathbf{0 . 3 8}$ & $\mathbf{2 3 . 0}$ & 0.29 & $\mathbf{1 0 . 6}$ & $\mathbf{0 . 1 8}$ \\
\hline
\end{tabular}

Table 1: Bloch simulation performance metrics comparing two purely spectral and two spectralspatial RF pulse designs for a phantom with paper clip susceptibility. The target flip angle $\alpha=19.6^{\circ}$ is approximately 0.34 radians. The best results are in bold.

\begin{tabular}{|l|l|l|l|l|l|}
\hline $\begin{array}{l}\text { Pulse Design } \\
\text { Trajectory }\end{array}$ & $\begin{array}{l}\text { Excitation } \\
\text { NRMSE }\end{array}$ & $\begin{array}{l}\text { Phase } \\
\text { RMSE }\left(^{\circ}\right)\end{array}$ & Mean Mag. & \% St. Dev & $\begin{array}{l}\text { Magnitude } \\
\text { NRMSE }\end{array}$ \\
\hline $\begin{array}{l}\text { Spectral } \\
\text { full BW }\end{array}$ & 0.54 & 25.2 & 0.17 & 19.6 & 0.39 \\
\hline $\begin{array}{l}\text { Spectral- } \\
\text { spatial 2 VDS }\end{array}$ & $\mathbf{0 . 1 8}$ & $\mathbf{7 . 0}$ & $\mathbf{0 . 2 4}$ & $\mathbf{7 . 7}$ & $\mathbf{0 . 1 5}$ \\
\hline
\end{tabular}

Table 2: Bloch simulation performance metrics comparing purely spectral and spectral-spatial RF pulses designed using a $2 \mathrm{D}$ slice of a human brain field map. The target flip angle $\alpha=15.8^{\circ}$ is approximately 0.28 radians. The best results are in bold.

Supporting Table S1: Possible combinations of design weighting matrices (columns) and simulation methods (rows) for spectral-spatial pulse design. The original method presented in the main paper is assigned the naming convention " $\mathbf{B} \mathbf{U}_{L} \mathbf{U}_{\text {std }}$ ".

Supporting Table S2: Performance metrics of all possible design weight matrix/simulation methods tested. The first row " $B \mathrm{U}_{L} \mathrm{U}_{\text {std }}$ " represents the weighting design matrix and simulation method used in the main paper. The bold values represent the best performance.

Supporting Table S3: Performance metrics defined in paper as (Eq. [7-11]) for a simulated hard pulse in comparison to purely spectral and spectral-spatial prewinding pulses. The bold values represent the best performance. 


\title{
Supporting Information: Design of spectral-spatial phase prewinding pulses and their use in small-tip fast recovery steady-state imaging
}

\author{
Sydney N. Williams ${ }^{1}$, Jon-Fredrik Nielsen ${ }^{1}$, Jeffrey A. Fessler ${ }^{2}$, Douglas C. Noll ${ }^{1}$ \\ 1. Department of Biomedical Engineering and 2. Department of Electrical Engineering and Computer \\ Science, University of Michigan, Ann Arbor, MI, USA
}

This document provides additional discussion and experimental results that supplement (1).

\section{A. Local spectral-spatial bandwidth as a model for intravoxel dephasing}

\section{Spatially varying bandwidth}

Choosing a local bandwidth $L$ to design spectral-spatial pulses faces multiple competing demands. On one hand, a wider bandwidth $L$ might balance the competing needs of spatial resolution and pulse length, allowing the prewinding pulse to capture larger range of off-resonance. On the other hand, a wider $L$ could increase SAR, making the RF optimization problem more challenging and lead to overall worse prewinding performance. This section explores a few design considerations for local bandwidth $L$ and concludes that simply using a uniform $L=25 \mathrm{~Hz}$ is sufficient for the spectral-spatial pulses designed in the paper.

One possible alternative is to adapt $L$ to changing $\Delta B_{0}$ offset based on (2), which noted that there is proportionality between off-resonance frequency $f(x, y)$ and through-plane gradient $g_{\mathrm{z}}$. This can be expressed as

$$
g_{\mathrm{z}}=\alpha f(x, y)
$$

where $\alpha$ is the proportionality constant. In (2), $\alpha$ was estimated from contiguous field maps as $-2.03 \times 10^{-4}$ $\mathrm{G} / \mathrm{cm} / \mathrm{Hz}$. We therefore chose to explore through-plane contributions to intravoxel bandwidth spread using $\alpha=-2 \times 10^{-4} \mathrm{G} / \mathrm{cm} / \mathrm{Hz}$. We did this by adapting the local bandwidth spatially considering through-plane gradient effects (2) by applying the following equation:

$$
L_{\mathrm{tp}}(x, y)=\gamma \alpha \Delta z|\Delta f(x, y)| \text {. }
$$

Here $L_{\mathrm{tp}}(x, y)$ is the spatially varying local bandwidth attributed to the through-plane gradient, $\gamma$ is the gyromagnetic ratio, and $\Delta z=0.4 \mathrm{~cm}$ was the slice thickness used in the 3D field map acquisition. We also centered the field map $\Delta f(x, y)$ to a median value of $0 \mathrm{~Hz}$. Using Eq. [S-2] alone would cause spatial locations at or close to the median field map frequency to have a through-plane local bandwidth value of about $0 \mathrm{~Hz}$. Furthermore, at the farthest field map deviation value of $129 \mathrm{~Hz}$, Eq. [S-2] would yield $L_{\mathrm{tp}}=44$ $\mathrm{Hz}$. We chose to balance the contribution of through-plane gradient and the original uniform $L=25 \mathrm{~Hz}$. We associate the $25 \mathrm{~Hz}$ bandwidth with microscopic effects of $\mathrm{T} 2 *$ decay, whereas the through-plane dephasing proportional to $\Delta B_{0}$ is related to macroscopic effects. These effects are largely independent and thus are treated as orthogonal sources of bandwidth spread. This leads to a quadrature combination, so the final spatially varying $L_{\text {total }}(x, y)$ we investigated is:

$$
L_{\text {total }}(x, y)=\sqrt{(\gamma \alpha \Delta z|\Delta f(x, y)|)^{2}+25^{2}} .
$$

We then incorporated this spatially varying bandwidth into the design through the weighting matrix. To 
design an RF pulse using Eq. [S-3], the weighting matrix W contained samples of

$$
w(x, y, f)= \begin{cases}1, & |f-\Delta f(x, y)| \leq \frac{L_{\text {total }}(x, y)}{2} \\ 0, & \text { otherwise }\end{cases}
$$

See Supp. Fig. S1 for an illustration that compares Eq. [S-4] (Supp. Fig. S1 bottom row) to the original fixed bandwidth design in Eq. [5] of (1) (Sup. Fig. S1 top row).

\section{Gaussian weights in the design weighting matrix}

These two implementations of $\mathbf{W}$ (spatially varying and uniform $L_{\text {total }}(x, y)$ ) are binary weighting matrices, where the only weight values used are 0's and 1's. We also investigated using Gaussian weights instead of binary weights in the design weighting matrix so it matches the Gaussian weight model used in calculating the performance metrics, e.g., Excitation NRMSE. In this case, the values of $w(x, y, f)$ included in the matrix are scaled to normalized Gaussian weights as

$$
w(x, y, f)= \begin{cases}\frac{g(f ; x, y)}{\max (g(f ; x, y))}, & |f-\Delta f(x, y)| \leq 3 L_{\text {total }}(x, y) \\ 0, & \text { otherwise }\end{cases}
$$

where $g(f ; x, y)$ is the Gaussian weight at frequency $f$. We define $g(f ; x, y)$ as

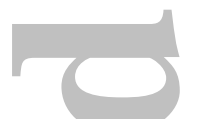

$$
g(f ; x, y)=\frac{1}{L_{\text {total }}(x, y) \sqrt{2 \pi}} \exp \left[-\frac{(\bar{f}(x, y)-f)^{2}}{2 L_{\text {total }}(x, y)^{2}}\right]
$$

where $\bar{f}(x, y)$ is the mean frequency within the $L_{\text {total }}(x, y)$ bandwidth region at location $(x, y)$ and the standard deviation $L_{\text {total }}(x, y)$ can also be uniform or spatially varying for the Gaussian weights.

In total, we investigated the design of spectral-spatial pulses with 4 unique weighting matrices W: binary entries (Eq. [S-4]) with i) uniform $L_{\text {total }}(x, y)=25 \mathrm{~Hz}$ intravoxel spread or ii) spatially varying spread $L_{\text {total }}(x, y)$ defined in Eq. [S-3], and Gaussian-weighted entries (Eq. [S-5]) with iii) uniform $L_{\text {total }}(x, y)=25$ $\mathrm{Hz}$ intravoxel spread and $25 \mathrm{~Hz}$ standard deviation or iv) spatially varying spread $L_{\text {total }}(x, y)$ and spatially varying standard deviation. Supporting Figure S1 illustrates these distinct design weighting matrices. Each box of Supp. Fig. S1 represents a sampled frequency $f$ and the plots are shown in spatial dimension $x, y$. To further investigate this approach, all simulation results use a finer spectral sampling rate of $\Delta f=5 \mathrm{~Hz}$ $(\Delta f=10 \mathrm{~Hz}$ was used in (1)).

\section{Spatially varying standard deviation in Bloch simulation}

For completeness, we also considered a refinement of the simulation methods. In (1), we used an intravoxel frequency spread in Bloch simulation spanning a $[-3 \sigma: 10: 3 \sigma] \mathrm{Hz}$ range, where $\sigma=25 \mathrm{~Hz}$ defined the standard deviation of normalized Gaussian distribution weights $\mathrm{g}$ used to combine these simulation results. Just like we considered spatially varying intravoxel widths for $\mathbf{W}$ of our spectral-spatial pulse design, we could simulate over ranges with spatially varying standard deviation $L_{\text {total }}(x, y)$ similar to Eq. [S-3]. Hence, we now have two methods for multi-frequency Bloch simulation: normalized Gaussian weights with a uniform standard deviation and a spatially varying standard deviation. 

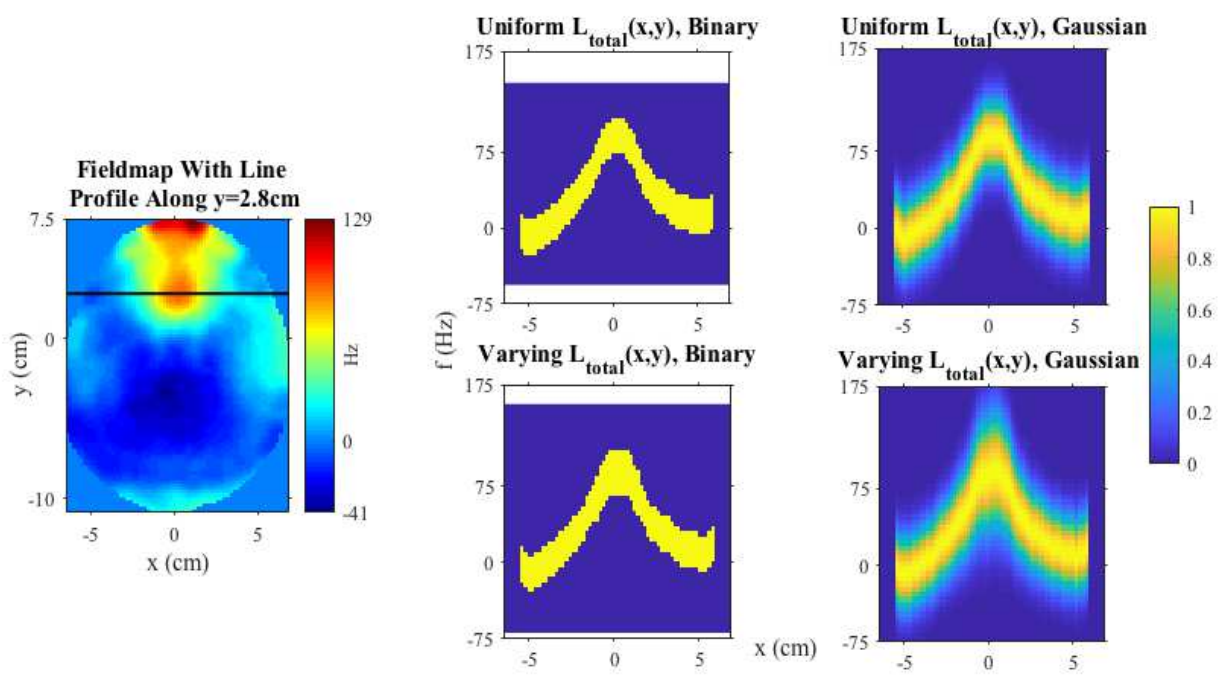

Supporting Figure S1: Four possible design weighting matrices $\mathbf{W}$ for spectral-spatial pulse prewinding. In the top row, the intravoxel bandwidth spread is universally $L_{\text {total }}(x, y)=25 \mathrm{~Hz}$ at all locations. In the bottom this value varies spatially with the spread proportional to through-plane gradient. In the left column, the values included in W are binary (0's or 1's). In the right column, the values are weighted by a normalized Gaussian distribution. In (1), we used the top left design weighting matrix.

\section{Results}

To facilitate our forthcoming discussion, Supporting Table S1 summarizes these design/simulation combinations and provides shorthand variable naming conventions for each possible combination of design matrix and simulation method.

\begin{tabular}{|c|l|l|l|l|l|}
\hline & & \multicolumn{4}{|c|}{ Design Weighting Matrix Method } \\
\hline \hline & & $\begin{array}{l}\text { Binary W with } \\
\text { Uniform } \\
L_{\text {total }}(x, y)\end{array}$ & $\begin{array}{l}\text { Binary W with } \\
\text { Varying } \\
L_{\text {total }}(x, y)\end{array}$ & $\begin{array}{l}\text { Gaussian W with } \\
\text { Uniform } \\
L_{\text {total }}(x, y)\end{array}$ & $\begin{array}{l}\text { Gaussian W with } \\
\text { Varying } \\
L_{\text {total }}(x, y)\end{array}$ \\
\hline $\begin{array}{l}\text { Simulation } \\
\text { Methods }\end{array}$ & $\begin{array}{l}\text { Gaussian with } \\
\text { Uniform } \\
\text { Standard } \\
\text { Deviation }\end{array}$ & $\mathbf{B U}_{L} \mathbf{U}_{\text {std }}$ & $\mathrm{BV}_{L} \mathrm{U}_{\text {std }}$ & $\mathrm{GU}_{L} \mathrm{U}_{\text {std }}$ & $\mathrm{GV}_{L} \mathrm{U}_{\text {std }}$ \\
\hline $\begin{array}{l}\text { Gaussian with } \\
\text { Varying } \\
\text { Standard } \\
\text { Deviation }\end{array}$ & $\mathrm{BU}_{L} \mathrm{~V}_{\text {std }}$ & $\mathrm{BV}_{L} \mathrm{~V}_{\text {std }}$ & $\mathrm{GU}_{L} \mathrm{~V}_{\text {std }}$ & $\mathrm{GV}_{L} \mathrm{~V}_{\text {std }}$ \\
\hline
\end{tabular}

Supporting Table S1: Possible combinations of design weighting matrices (columns) and simulation methods (rows) for spectral-spatial pulse design. The original method presented in the main paper is assigned the naming convention "B $\mathbf{U}_{L} \mathbf{U}_{\text {std" }}$ ".

We explored designing four sets of spectral-spatial pulses (using four weighting matrix methods) and simulated their performance with both simulation methods for the fieldmap shown in Supp. Fig. S1. One of these combinations, "BU $\mathrm{U}_{\text {std", }}$, is used in (1) (albeit with $10 \mathrm{~Hz}$ rather than $5 \mathrm{~Hz}$ spacing for the design). Supporting Table S2 lists the performance of all 8 design/simulation combinations with the naming conventions provided in Supporting Table S1. 


\begin{tabular}{|l|l|l|l|l|l|}
\hline $\begin{array}{l}\text { Design/ } \\
\text { Simulation } \\
\text { Method }\end{array}$ & $\begin{array}{l}\text { Excitation } \\
\text { NRMSE }\end{array}$ & $\begin{array}{l}\text { Phase } \\
\text { RMSE }\left(^{\circ}\right)\end{array}$ & $\begin{array}{l}\text { Mean } \\
\text { Magnitude }\end{array}$ & $\begin{array}{l}\text { \% Magnitude } \\
\text { St. Dev. }\end{array}$ & $\begin{array}{l}\text { Magnitude } \\
\text { NRMSE }\end{array}$ \\
\hline \hline $\mathrm{BU}_{L} \mathrm{U}_{\text {std }}$ & $\mathbf{0 . 1 8}$ & 7.4 & $\mathbf{0 . 2 4}$ & 7.8 & $\mathbf{0 . 1 4}$ \\
\hline $\mathrm{BV}_{L} \mathrm{U}_{\text {std }}$ & $\mathbf{0 . 1 8}$ & $\mathbf{7 . 0}$ & $\mathbf{0 . 2 4}$ & $\mathbf{7 . 0}$ & $\mathbf{0 . 1 4}$ \\
\hline $\mathrm{GU}_{L} \mathrm{U}_{\text {std }}$ & 0.29 & 8.0 & 0.20 & 7.8 & 0.26 \\
\hline $\mathrm{GV}_{L} \mathrm{U}_{\text {std }}$ & 0.32 & 8.1 & 0.20 & 11.1 & 0.31 \\
\hline $\mathrm{BU}_{L} \mathrm{~V}_{\text {std }}$ & 0.21 & 8.8 & $\mathbf{0 . 2 4}$ & 11.4 & 0.17 \\
\hline $\mathrm{BV}_{L} \mathrm{~V}_{\text {std }}$ & 0.21 & 8.0 & $\mathbf{0 . 2 4}$ & 9.9 & 0.17 \\
\hline $\mathrm{GU}_{L} \mathrm{~V}_{\text {std }}$ & 0.31 & 9.8 & 0.20 & 10.4 & 0.28 \\
\hline $\mathrm{GV}_{L} \mathrm{~V}_{\text {std }}$ & 0.33 & 9.3 & 0.20 & 12.5 & 0.31 \\
\hline
\end{tabular}

Supporting Table S2: Performance metrics of all possible design weight matrix/simulation methods tested. The first row " $B U_{L} \mathrm{U}_{\text {std }}$ " represents the weighting design matrix and simulation method used in (1). The bold values represent the best performance.

We expected the performance metrics, particularly excitation NRMSE and phase RMSE, to worsen when simulation methods went from using a Gaussian spread of intravoxel frequencies with uniform standard deviation $\sigma$ to a spatially varying spread where $\sigma$ increased at areas of higher off-resonance due to throughplane effects. Supporting Table S2 shows where performance drops slightly from design/simulation combinations ending in " $\mathrm{XXU}_{\text {std }}$ " to " $\mathrm{XXV}_{\text {std" }}$ ". It was harder to predict how adjusting the design weighting matrix would affect the spectral-spatial pulse performance. Supporting Table S2 reports decreases in performance for design weighting matrices when adjusting from binary to Gaussian weight values ("BXX" to "GXX"). There are negligible differences when comparing uniform and varying $L_{\text {total }}(x, y)$ in the weight matrix (" $\mathrm{X} U_{L} X$ " to " $\mathrm{X} V_{L} \mathrm{X}$ "). This is likely because changing the design weight matrix from binary to Gaussian weights increases the support of $\mathbf{W}$ in the frequency dimension, resulting in a more challenging RF design. The magnitude simulation images (Supp. Fig. S2) and phase simulation images (Supp. Fig. S3) are visually consistent with the fact that the various design alternatives yield similar results but with a slight decline for some explored methods for this particular slice.

In conclusion, we explored modifying the weighting matrix used in designing spectral-spatial pulses by i) varying the design target bandwidth $L_{\text {total }}(x, y)$ to reflect the proportionality between off-resonance and through-plane gradient using references such as (2) and ii) varying the weights of the design weighting matrix to be Gaussian rather than binary. We also went further and investigated simulation methods where we varied the standard deviation of the Gaussian intravoxel spread of frequencies to be spatially varying with off-resonance. After testing all possible combinations, we saw only small changes in simulation performance metrics. It is possible that with higher spatial resolution excitation k-space trajectories we might see greater gains with these modifications. However, this would also mean a longer RF pulse, so the potential advantages of these changes is unclear. 


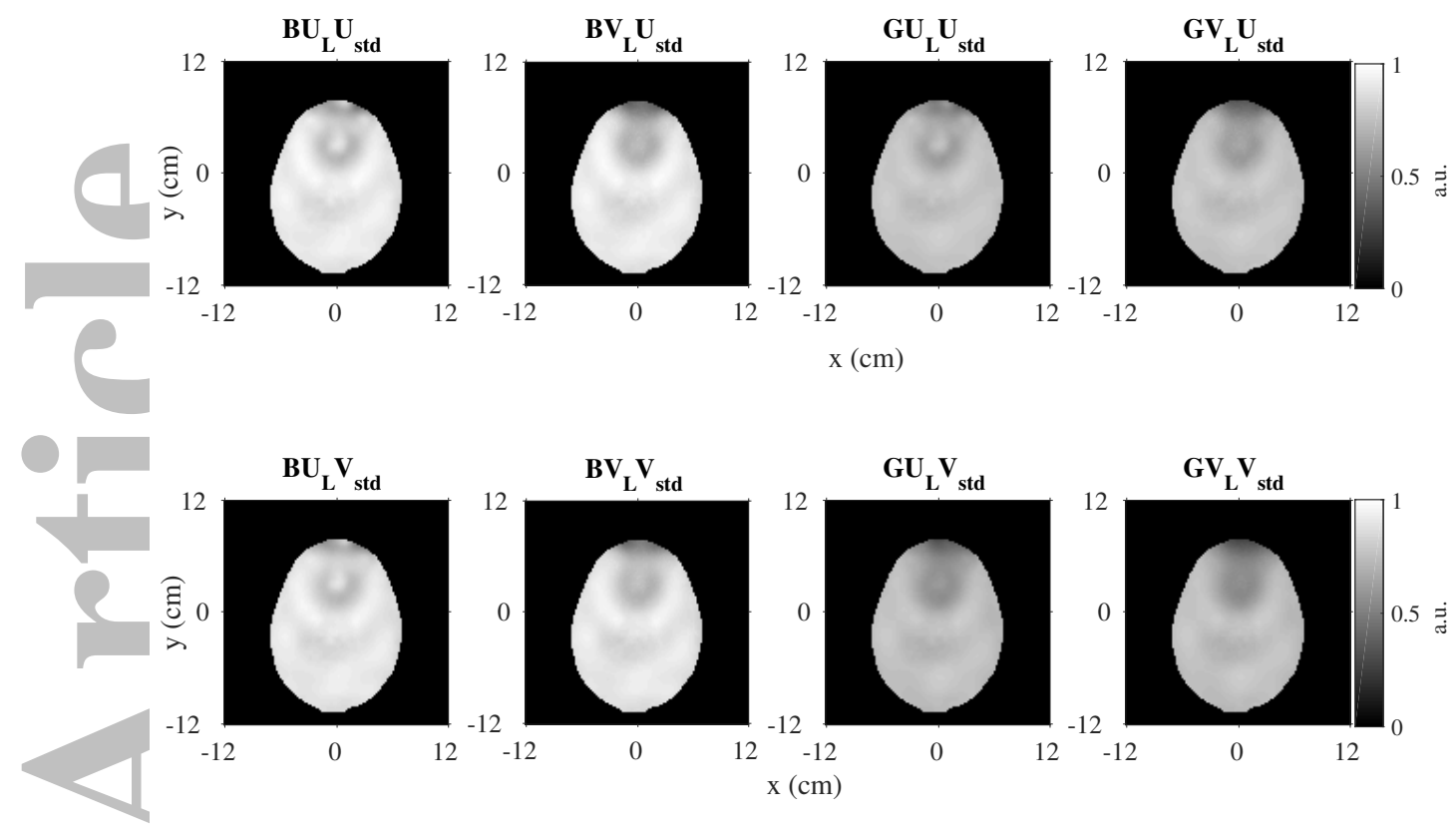

Supporting Figure S2: Simulated magnitude images for all possible design weighting matrix and simulation combinations presented in Table S1.

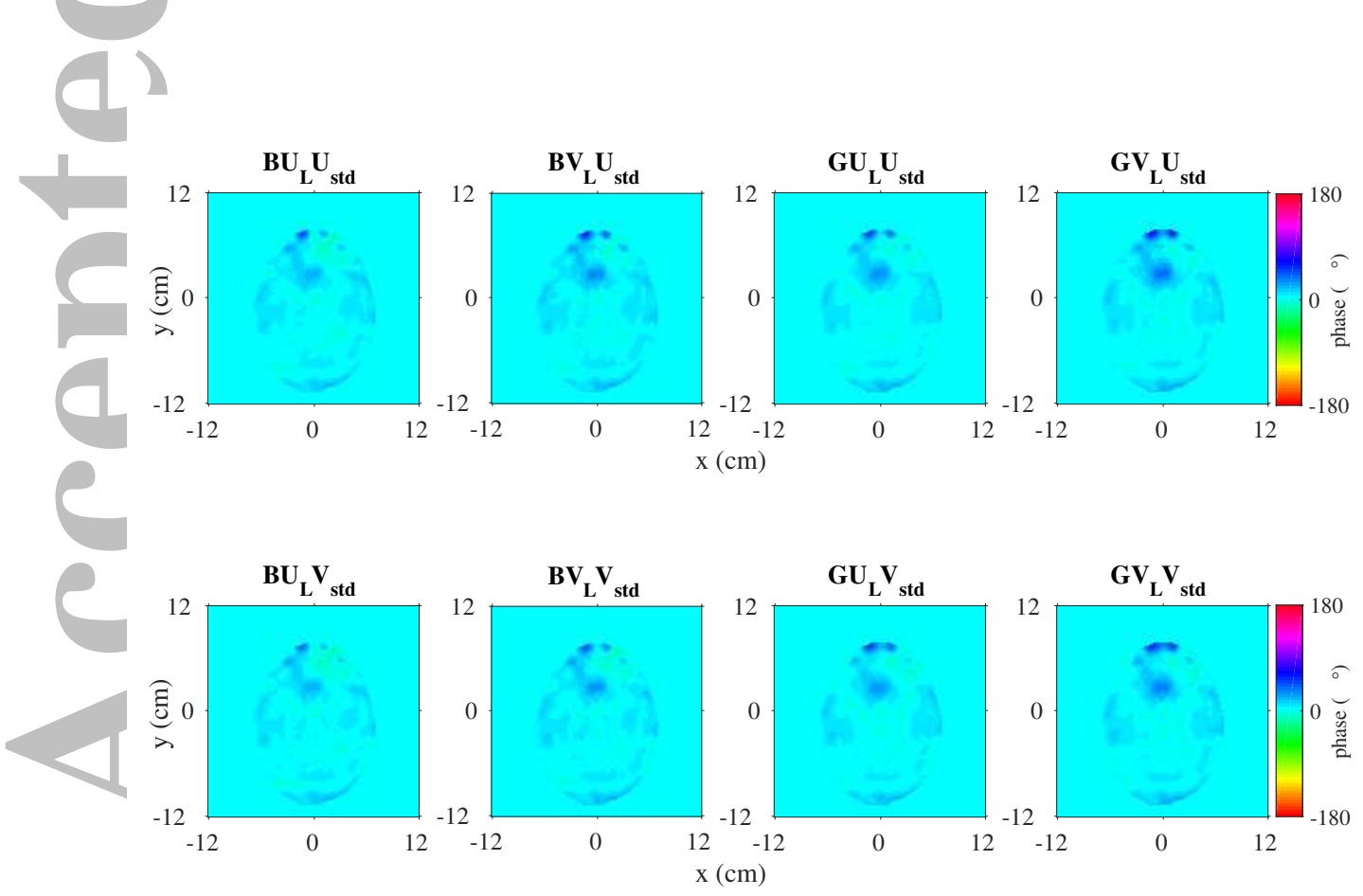

Supporting Figure S3: Simulated phase images for all possible design weighting matrix and simulation combinations presented in Table S1. 


\section{B. Hard pulse simulation and prewinding pulse performance over one fre- quency and one spatial dimension}

In (1) we compare spectral and spectral-spatial prewinding pulses, building on previous work in (3) and (4). In this supporting information, we also compare these to the performance of a non-prewinding pulse. In this case, we simulate a simple $500 \mu$ s hard rect pulse that has the same TE and flip angle as our human spectral and spectral-spatial RF pulse designs $\left(3.648 \mathrm{~ms} / 16^{\circ}\right)$. Supporting Table S3 below reports performance metrics (Eq. [7-11] in main paper) for this hard pulse and the spectral and spectral-spatial pulses. As expected, the hard pulse has a uniform magnetization appearance but does not reach the target magnitude ( $\%$ magnitude standard deviation, magnitude NRMSE) and fails to acheive a flat phase profile since no prewinding has occured (phase RMSE, excitation NRMSE).

\begin{tabular}{|l|l|l|l|l|l|}
\hline Pulse & $\begin{array}{l}\text { Excitation } \\
\text { NRMSE }\end{array}$ & $\begin{array}{l}\text { Phase } \\
\text { RMSE }\left({ }^{\circ}\right)\end{array}$ & $\begin{array}{l}\text { Mean } \\
\text { Magnitude }\end{array}$ & $\begin{array}{l}\% \text { Magnitude } \\
\text { St. Dev. }\end{array}$ & $\begin{array}{l}\text { Magnitude } \\
\text { NRMSE }\end{array}$ \\
\hline \hline Hard pulse & 0.64 & 43.5 & $\mathbf{0 . 2 4}$ & $\mathbf{0 . 1}$ & $\mathbf{0 . 1 3}$ \\
\hline $\begin{array}{l}\text { Purely spectral } \\
\text { pulse }\end{array}$ & 0.54 & 25.2 & 0.17 & 19.6 & 0.39 \\
\hline $\begin{array}{l}\text { Spectral-spatial } \\
\text { pulse }\end{array}$ & $\mathbf{0 . 1 8}$ & $\mathbf{7 . 0}$ & $\mathbf{0 . 2 4}$ & 7.7 & 0.15 \\
\hline
\end{tabular}

Supporting Table S3: Performance metrics defined in paper as (Eq. [7-11]) for a simulated hard pulse in comparison to purely spectral and spectral-spatial prewinding pulses. The bold values represent the best performance.

In addition to the performance metrics provided, we have created plots to demonstrate the performance of prewinding pulses as a function of one spatial dimension and frequency before summation using Gaussian weights. To do this, we selected a particular " $y$ " location in the 2D in vivo field map and drew a line profile spanning all " $x$ " through it. We then examined the magnetization of both purely spectral and spectral-spatial pulses across this line profile for all frequencies included in Bloch simulation. Finally, we repeated this same process for one " $x$ " location and the corresponding " $y$ " line profile.

Supporting Figure S4 shows the magnetization simulations for one spatial dimension and all simulation frequencies for the hard pulse. The simulations are presented in terms of relative complex error $\left(\left|\frac{m_{x y}}{\sin \alpha}-1\right|\right)$, absolute phase error $\left(\left|\angle m_{x y}\right|\right)$, and relative magnitude error $\left(\left|\frac{\left|m_{x y}\right|}{\sin \alpha}-1\right|\right)$. This diagram also shows the 2D human field map, simulated magnitude, and simulated phase over all combined frequencies using a Gaussian distribution with $\sigma=25 \mathrm{~Hz}$ as described in the main paper. Supporting Figures S5 and S6 repeat these plots for the purely spectral and spectral-spatial pulse, respectively. 

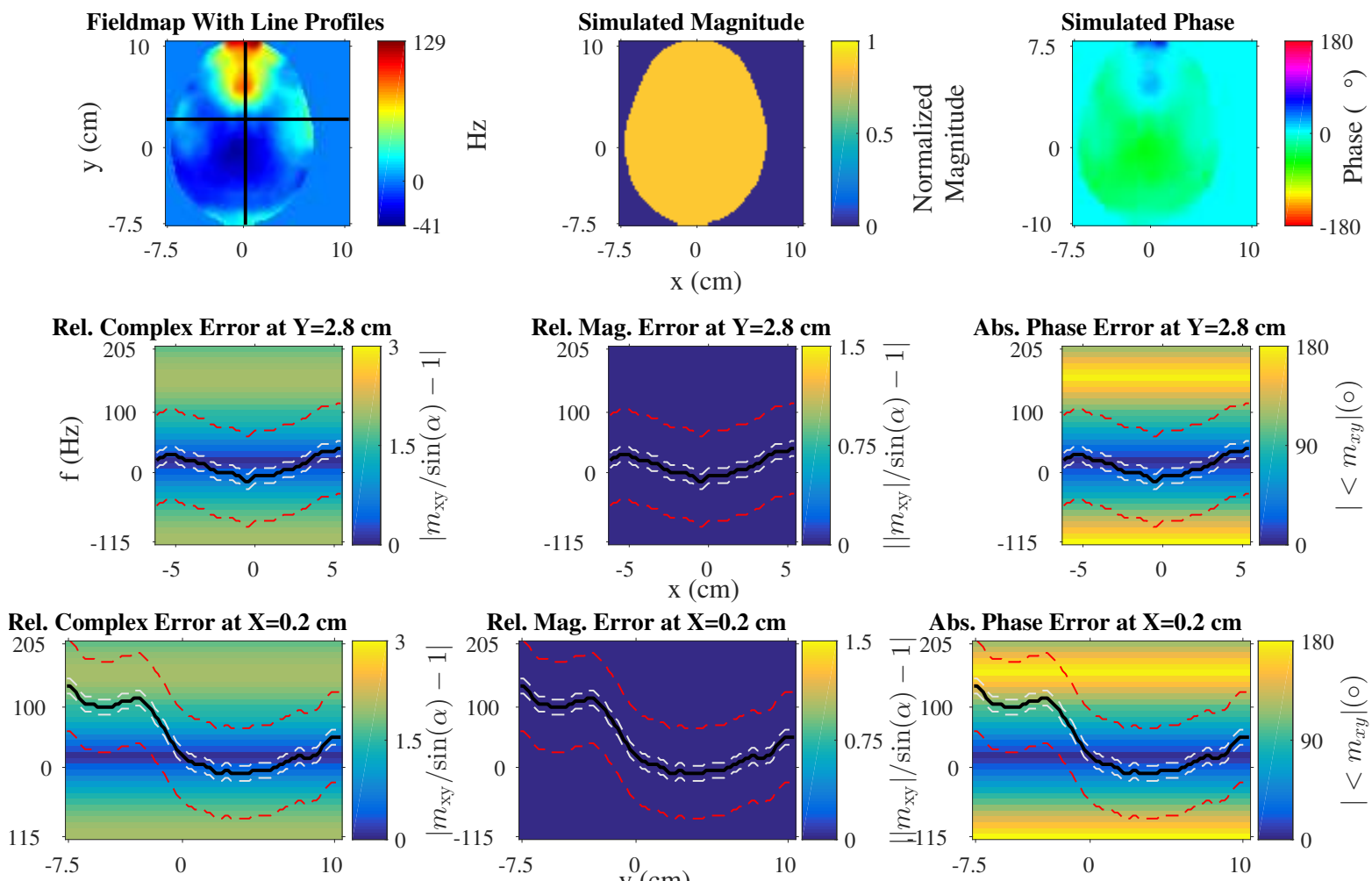

Rel. Mag. Error at $\mathrm{X}=0.2 \mathrm{~cm}$

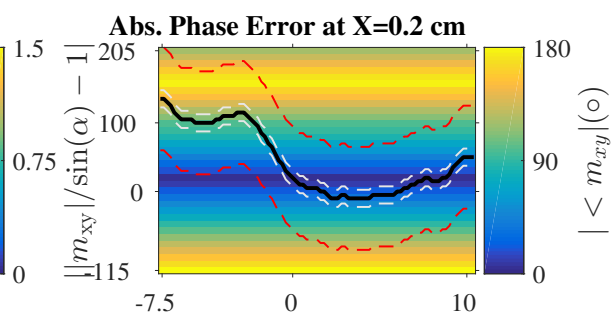

Supporting Figure S4: Hard pulse simulation results. Top row: 2D field map (left), simulated magnitude (center), and simulated phase (right). Middle row: relative complex error, relative magnitude error, and absolute phase error for the $1 \mathrm{D}$ line profile magnitude across all $x$ at $y=2.8 \mathrm{~cm}$. Bottom row: relative complex error, relative magnitude error, and absolute phase error for profile across all $y$ at $y=0.2 \mathrm{~cm}$. The dashed white lines represent the $L=25 \mathrm{~Hz}$ used as the local bandwidth for the spectral spatial pulse, and the dashed red lines represent the $\pm 3 \sigma=75 \mathrm{~Hz}$ bandwidth used in Bloch simulation. These plots are repeated for the spectral and spectral-spatial pulses in Supp. Figs S5 and S6. 

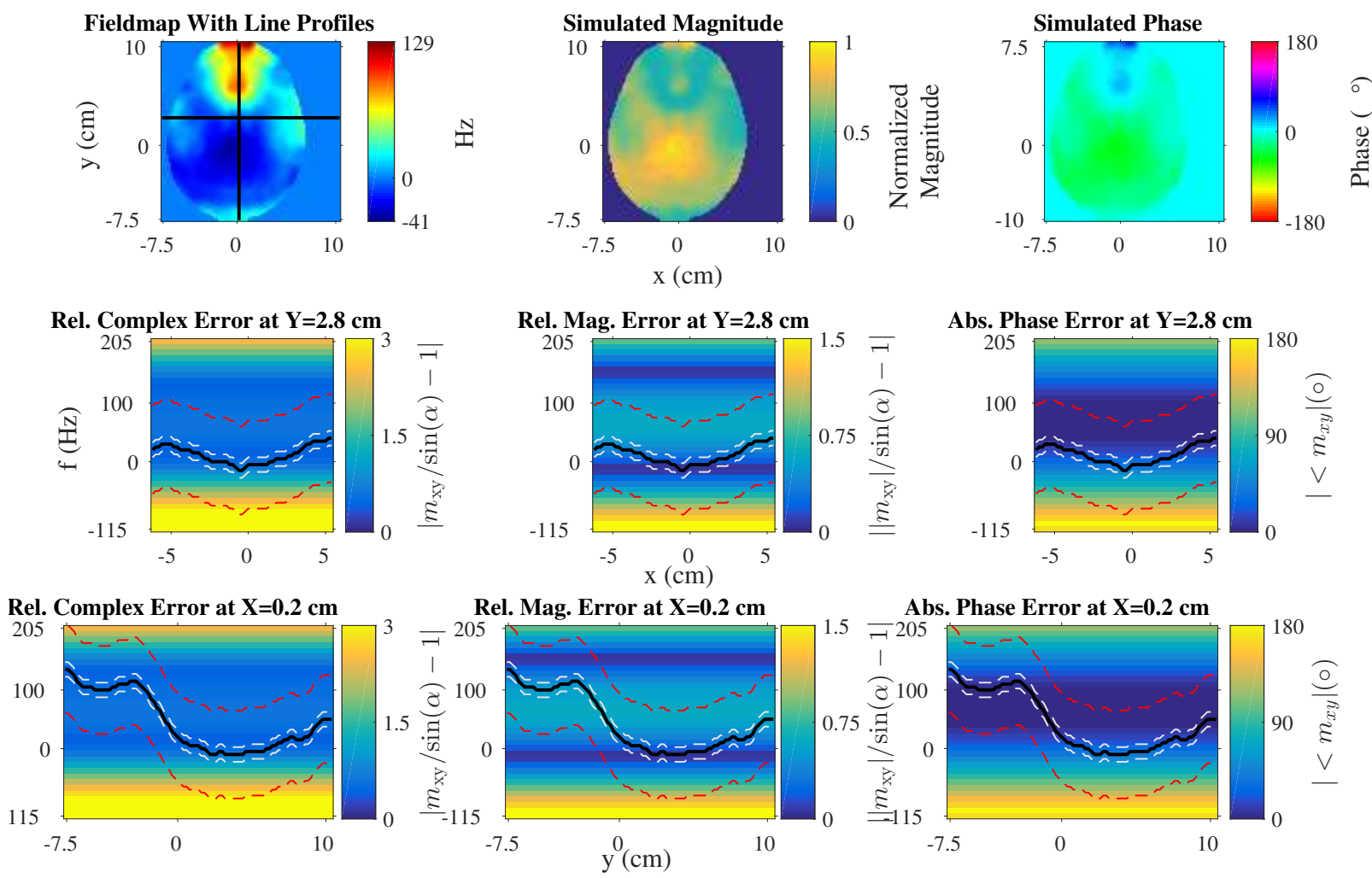

Supporting Figure S5: Purely spectral pulse simulation results. Compare with Supp. Figs S4 and S6.

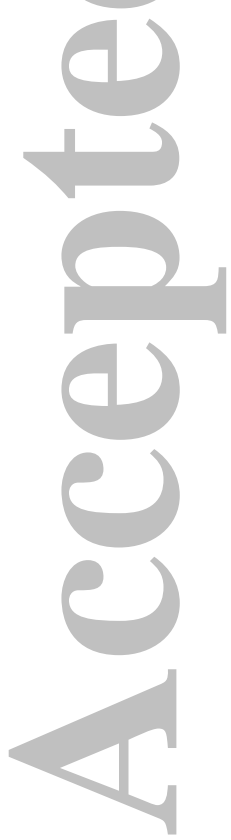



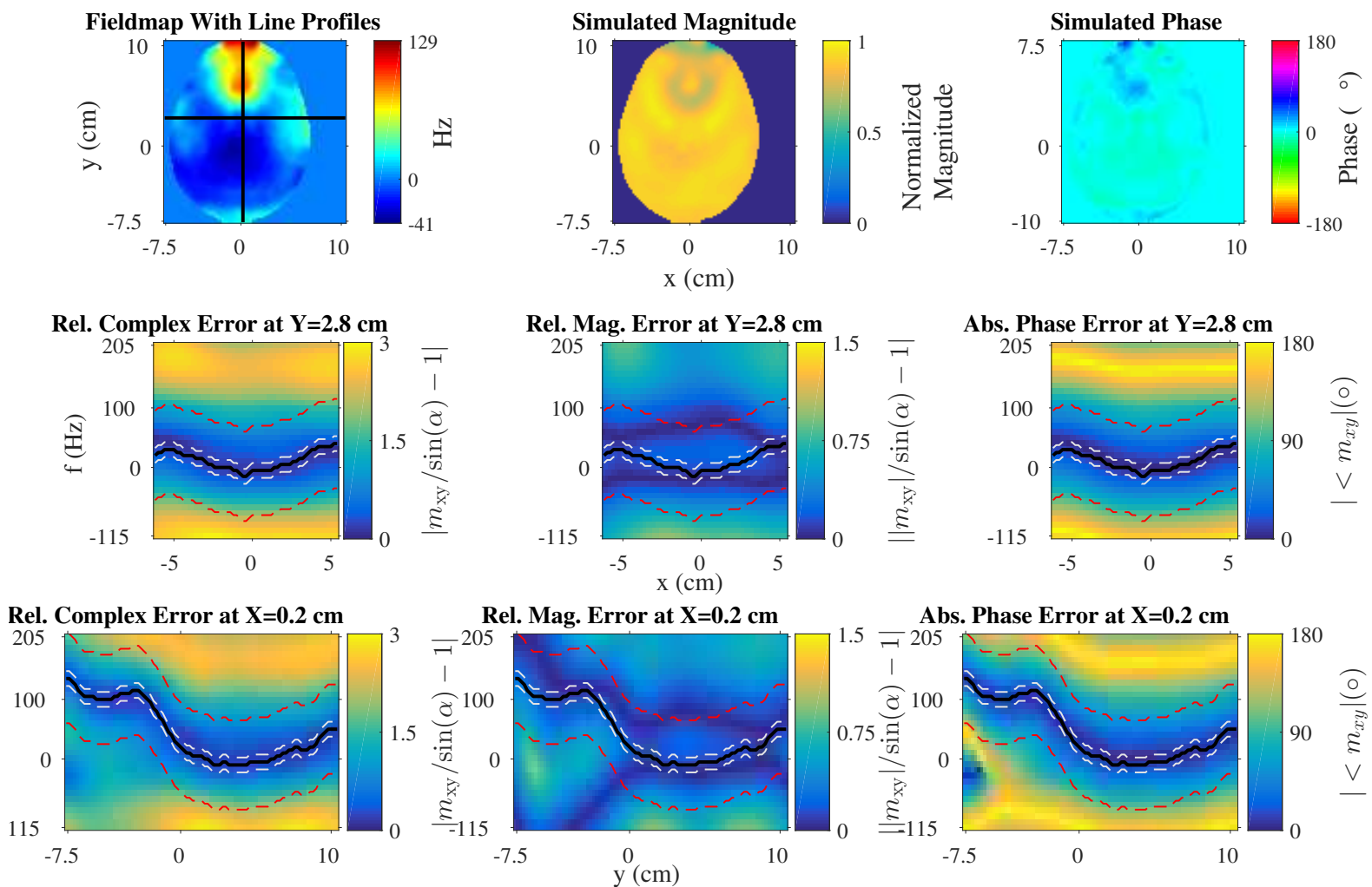

Abs. Phase Error at $\mathrm{X}=0.2 \mathrm{~cm}$

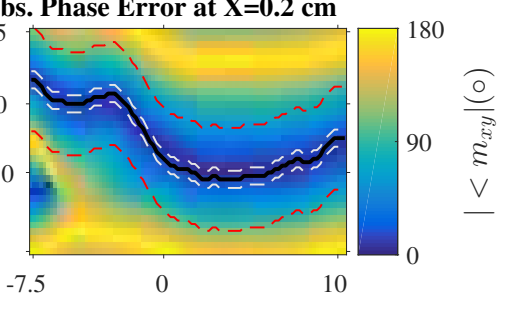

Supporting Figure S6: Spectral-spatial pulse simulation results. Compare with Supp. Figs S4 and S5. 
The hard pulse plots in Supp. Fig. S4 agree with the performance metrics in Supporting Table S3 in that the pulse performs poorly with large absolute phase error and relative magnitude and complex error across all space. As anticipated, the spectral-spatial pulse in Supp. Fig. S6 tracks the spatially varying off-resonance while the purely spectral pulse in Supp. Fig. S5 does not. The purely spectral pulse has low phase error values for some spatial locations, but with varying off-resonance it cannot enforce low relative complex at all frequencies. Meanwhile, the spectral-spatial pulse maintains particularly low relative complex error with spatial variation. We therefore conclude that both effective magnitude and phase performance (in other words, the relative complex error) is what leads to effective prewinding. The summed frequency simulation of these three pulses in the 2D simulated magnitude and phase images are consistent with these performance differences as well.

Additional intuition about pulse performance can be gained by plotting "error" as a function of spatial location. Figure 7 in the main paper shows excitation and phase root squared error combined across all simulated frequencies as a function of spatial location for the purely spectral pulse and spectral-spatial pulse. There, we can easily see that the hard pulse performs very poorly, the spectral pulse performs moderately well, and the spectral-spatial pulse performs has the lowest phase errors.

\section{References}

[1] Williams SN, Nielsen J-F, Fessler JA, and Noll DC. Design of spectral-spatial phase prewinding pulses and their use in small-tip fast recovery steady-state imaging Magn Res Med (submitted).

[2] Yip C, Yoon D, Olafsson V, Lee S, Grissom WA, Fessler JA, Noll DC. Spectral-spatial pulse design for through-plane phase precompensatory slice selection in $\mathrm{T}_{2}^{*}$-weighted functional MRI. Magn Res Med 2009;61:1137-47.

[3] Assländer J, Glaser SJ, Hennig J. Spin echoes in the regime of weak dephasing. Magn Res Med 2016; 75:150-160.

[4] Sun H, Fessler JA, Noll DC, Nielsen JF. Balanced SSFP-like steady-state imaging using small-tip fast recovery (STFR) sequence with a spectral pre-winding pulse. Magn Res Med 2016;75:839-844. 